\title{
GROUP-VALUED EQUIVARIANT LOCALIZATION
}

\author{
A. ALEKSEEV, E. MEINRENKEN, AND C.WOODWARD
}

\begin{abstract}
We prove a localization formula for group-valued equivariant de Rham cohomology of a compact $G$-manifold. This formula is a non-trivial generalization of the localization formula of Berline-Vergne and Atiyah-Bott for the usual equivariant de Rham cohomology. As an application, we obtain a version of the Duistermaat-Heckman formula for Hamiltonian spaces with group-valued moment maps.
\end{abstract}

\section{INTRODUCTION}

The main result of this paper is a localization theorem for $G$-manifolds $M$ which we view as the group valued analogue of the localization formula (Berline-Vergne [6], Atiyah-Bott [4]) in equivariant de Rham theory.

Recall that the equivariant cohomology of $M$ (over $\mathbb{C}$ ) is the cohomology of Cartan's [9] complex $C_{G}(M)=\left(S \mathfrak{g}_{\mathbb{C}}^{*} \otimes \Omega(M)\right)^{G}$ of equivariant differential forms, equipped with a certain differential $\mathrm{d}_{G}$. The complex $C_{G}(M)$ embeds into the larger complex complex $\widehat{C}_{G}(M)=\left(\mathcal{E}^{\prime}\left(\mathfrak{g}^{*}\right) \otimes \Omega(M)\right)^{G}$ where $\mathcal{E}^{\prime}\left(\mathfrak{g}^{*}\right)$ is the convolution algebra of compactly supported distributions, and $S \mathfrak{g}^{*}$ is viewed as distributions supported at 0 . If $M$ is compact and oriented, integration over $M$ defines a chain map

$$
\int_{M}: \widehat{C}_{G}(M) \rightarrow \widehat{C}_{G}(\mathrm{pt})=\mathcal{E}^{\prime}\left(\mathfrak{g}^{*}\right)^{G} .
$$

For any cocycle $\beta \in \widehat{C}_{G}(M)$, the localization formula expresses the value of the Fourier transform $\left\langle\int_{M} \beta, e^{2 \pi i\langle, \xi\rangle}\right\rangle$ in terms of integrals over the connected components $F$ of the zero set of the vector field $\xi_{M}$ generated by $\xi$ :

$$
\left\langle\int_{M} \beta, e^{2 \pi i<, \xi\rangle}\right\rangle=\sum_{F \in \mathcal{F}(\xi)} \int_{F} \frac{\iota_{F}^{*}\left\langle\beta, e^{2 \pi i<\cdot \xi\rangle}\right\rangle}{\operatorname{Eul}\left(\nu_{F}, 2 \pi i \xi\right)} .
$$

Here $\operatorname{Eul}\left(\nu_{F}, 2 \pi i \xi\right)$ denotes the equivariant Euler form of the normal bundle. A special case of the localization theorem is the Duistermaat-Heckman "exact stationary phase" formula from symplectic geometry, in which $\beta$ is the equivariant Liouville form.

In [2] we introduced a new version of the equivariant de Rham complex where the dual of the Lie algebra $\mathfrak{g}^{*}$ is replaced by the Lie group $G$. That is, the chain complex is the space $\widehat{\mathcal{C}}_{G}(M)=\left(\mathcal{E}^{\prime}(G) \otimes \Omega(M)\right)^{G}$, with a suitable differential $\mathrm{d}_{G}$ depending on the

Date: May 1999. 
choice of an invariant inner product on $\mathfrak{g}$. Integration is a chain map

$$
\int_{M}: \widehat{\mathcal{C}}_{G}(M) \rightarrow \widehat{\mathcal{C}}_{G}(\mathrm{pt})=\mathcal{E}^{\prime}(G)^{G}
$$

Our localization formula describes the Fourier coefficients of the integral $\int_{M} \beta$ of any equivariant cocycle, that is, its pairings with irreducible characters of $G$. Let $T$ be a maximal torus of $G$, with Lie algebra $\mathfrak{t}$, and let $\lambda \in \mathfrak{t}^{*}$ be a dominant weight for some choice of positive Weyl chamber. It parametrizes an irreducible representation $V_{\lambda}$, with character $\chi_{\lambda}$. Let $\rho \in \mathfrak{t}^{*}$ be the half-sum of positive roots and use the inner product to identify $\mathfrak{t}^{*} \cong \mathfrak{t}$. Our main result expresses the Fourier coefficient $\left\langle\int_{M} \beta, \chi_{\lambda}\right\rangle$ in terms of integrals over connected components $F$ of the zero set of the vector field $(\lambda+\rho)_{M}$, as follows.

$$
\left\langle\int_{M} \beta, \chi_{\lambda}\right\rangle=\operatorname{dim} V_{\lambda} \sum_{F \in \mathcal{F}(\lambda+\rho)} \int_{F} \frac{\iota_{F}^{*}\left(e^{\frac{1}{2} \iota\left(r_{M}\right)}\left\langle\beta, \Delta_{\lambda}\right\rangle\right)}{\operatorname{Eul}\left(\nu_{F}, 2 \pi i(\lambda+\rho)\right)} .
$$

It involves the bi-vector field $r_{M}$ defined by the classical $r$-matrix, and certain "spherical harmonics" $\Delta_{\lambda} \in C^{\infty}(G)$ determined by $\lambda$.

There are several conceptual differences between the localization formulas (1) and (2). In (11) the equivariant parameter $\xi$ varies continuously, and for an open dense set of $\xi \in \mathfrak{t}$ the fixed point set is just the fixed point set for the maximal torus. It follows by continuity that if the maximal torus has no fixed points then the integral of an equivariant cocycle vanishes. The situation in (2) is different because $\lambda$ only varies in a discrete set. Hence the continuity argument does not apply, and integrals of equivariant cocycles may be non-zero even if the maximal torus has no fixed points. Next, for $\xi=0$ the localization formula (11) becomes tautological, whereas (2) gives a non-trivial result even for the $\lambda=0$ Fourier coefficient, which localizes to the set of zeroes of $\rho_{M}$.

We use the new localization theorem to obtain Duistermaat-Heckman type formulas for the theory of group-valued moment maps [1, 3]. In this case the localization contributions simplify and lead to formulas for intersection pairings on reduced spaces.

This result applies in particular to intersection pairings on moduli spaces of flat $G$ connections on surfaces. According to [1, 3] these spaces are reductions of group valued Hamiltonian $G$-spaces. In this example, our localization formula produces the formulas conjectured by Witten in [19] and confirmed (for $G=\mathrm{SU}(n)$ ) by Jeffrey-Kirwan [14]. Details of the application to moduli spaces will be presented in a forthcoming paper.

The paper is organized as follows. Section 2 is a review of equivariant de Rham theory. In particular, we describe the "group valued" version introduced in [2]. In Section 3 we construct a suitable "restriction map" from $G$-valued equivariant cohomology to $T$-valued equivariant cohomology, and in Section 1 we discuss some of its properties. This "abelianization" procedure is the key step in our proof of the $G$-valued localization formula in Section 5. In Section 6 we use the localization formula to prove a DuistermaatHeckman principle for group valued moment maps. 


\section{Equivariant DE Rham THEORY}

In this Section we recall the Cartan and Weil models of equivariant de Rham theory, and their non-commutative versions introduced in [2]. For a more detailed account of equivariant de Rham theory see e.g. the forthcoming book of Guillemin-Sternberg [12].

Throughout this paper, $G$ will denote a compact, connected Lie group with Lie algebra $\mathfrak{g}$. (Occasionally we will make some additional assumptions on $G$.)

For any manifold $M$ we denote by $\Omega^{\star}(M)$ the de Rham complex of complex-valued differential forms. Given a $G$-action on $M$, let

$$
\xi_{M}=\left.\frac{\mathrm{d}}{\mathrm{d} t}\right|_{t=0} \exp (-t \xi)^{*}
$$

denote the fundamental vector field corresponding to $\xi \in \mathfrak{g}$. Let

$$
L_{\xi}: \Omega^{\star}(M) \rightarrow \Omega^{\star}(M), \quad \iota_{\xi}: \Omega^{\star}(M) \rightarrow \Omega^{\star-1}(M)
$$

be the Lie derivative (resp. contraction) by $\xi_{M}$. Given a basis $\left\{e_{a}\right\} \subset \mathfrak{g}$ we also use the notation $L_{a}=L_{e_{a}}$ and $\iota_{a}=\iota_{e_{a}}$.

\subsection{Cartan models.}

2.1.1. Equivariant cohomology. Let $\mathcal{E}^{\prime}\left(\mathfrak{g}^{*}\right)$ be the convolution algebra of compactly supported complex-valued distributions on $\mathfrak{g}^{*}$. The subalgebra of distributions supported at 0 is identified with the symmetric algebra $S \mathfrak{g}_{\mathbb{C}}^{*}$, which is generated by the elements

$$
v^{a}=\left.\frac{\mathrm{d}}{\mathrm{d} t} \delta_{t e^{a}}\right|_{t=0}=-\frac{\partial}{\partial \mu_{a}} \delta_{0}
$$

corresponding to the dual basis vectors $e^{a}$. Let

$$
C_{G}(M)=\left(S \mathfrak{g}_{\mathbb{C}}^{*} \otimes \Omega(M)\right)^{G}, \quad \widehat{C}_{G}(M)=\left(\mathcal{E}^{\prime}\left(\mathfrak{g}^{*}\right) \otimes \Omega(M)\right)^{G}
$$

be the space of equivariant differential forms (resp. with distributional coefficients.) The derivation

$$
\mathrm{d}_{G}=1 \otimes \mathrm{d}-v^{a} \otimes \iota_{a}
$$

(using summation convention) of $C_{G}(M), \widehat{C}_{G}(M)$ squares to 0 , and defines the equivariant cohomologies $H_{G}(M), \widehat{H}_{G}(M)$. Both $H_{G}$ and $\widehat{H}_{G}$ are functorial with respect to maps between $G$-manifolds. In particular, the map $M \rightarrow$ pt makes $H_{G}(M)$ into a module over the ring $H_{G}(\mathrm{pt})=\left(S \mathfrak{g}_{\mathbb{C}}^{*}\right)^{G}$ of invariant polynomials and $\widehat{H}_{G}(M)$ into a module over the ring $\widehat{H}_{G}(\mathrm{pt})=\mathcal{E}^{\prime}\left(\mathfrak{g}^{*}\right)^{G}$ of invariant compactly supported distributions.

2.1.2. G-valued equivariant cohomology. In [2] the following non-commutative Cartan complexes $\mathcal{C}_{G}(M)$ and $\widehat{\mathcal{C}}_{G}(M)$ were introduced. Let $\mathcal{E}^{\prime}(G)$ be the convolution algebra of complex-valued distributions on the group $G$. The subalgebra of distributions supported at the group unit is isomorphic to the universal enveloping algebra $U(\mathfrak{g})_{\mathbb{C}}$, with

$$
u_{a}=\left.\frac{\mathrm{d}}{\mathrm{d} t} \delta_{\exp \left(t e_{a}\right)}\right|_{t=0}
$$


representing the generator corresponding to the basis $e_{a}$. Let

$$
\mathcal{C}_{G}(M)=\left(U(\mathfrak{g})_{\mathbb{C}} \otimes \Omega(M)\right)^{G}, \quad \widehat{\mathcal{C}}_{G}(M)=\left(\mathcal{E}^{\prime}(G) \otimes \Omega(M)\right)^{G} .
$$

To define a differential on $\mathcal{C}_{G}(M), \widehat{\mathcal{C}}_{G}(M)$ we assume that $\mathfrak{g}$ comes equipped with an invariant inner product, and take the basis $e_{a}$ to be orthonormal. One finds that

$$
\mathrm{d}_{G}=1 \otimes \mathrm{d}-\frac{u_{a}^{L}+u_{a}^{R}}{2} \otimes \iota_{a}+\frac{1}{24} f_{a b c} 1 \otimes \iota_{a} \iota_{b} \iota_{c}
$$

(using summation convention) is a differential, i.e. $\mathrm{d}_{G} \circ \mathrm{d}_{G}=0$. Here $f_{a b c}=\left[e_{a}, e_{b}\right] \cdot e_{c}$ are the structure constants for the given basis and $u_{a}^{L}, u_{a}^{R}$ denote the operators of left-/right multiplication (that is, convolution) by $u_{a}$. We denote the corresponding cohomologies by $\mathcal{H}_{G}(M), \widehat{\mathcal{H}}_{G}(M)$. Notice that $\mathrm{d}_{G}$ is not a derivation with respect to the obvious ring structure on $\widehat{\mathcal{C}}_{G}(M)$. However, one can modify the ring structure as follows: Let $\Omega(M)$ be equipped with the non-(super-)commutative ring structure defined by

$$
\beta_{1} \odot \beta_{2}=\operatorname{diag}_{M}^{*} \circ \exp \left(-\frac{1}{2} \iota_{a}^{1} \iota_{a}^{2}\right)\left(\beta_{1} \times \beta_{2}\right)
$$

where $\operatorname{diag}_{M}^{*}$ is pull-back by the diagonal embedding, and $\iota_{a}^{j}$ are contractions acting on the $j$ th factor. Together with the natural ring structure on $U(\mathfrak{g}), \mathcal{E}^{\prime}(G)$, it defines a ring structure $\odot$ on $\mathcal{C}_{G}(M), \widehat{\mathcal{C}}_{G}(M)$ for which $\mathrm{d}_{G}$ is a derivation. In this way the cohomologies $\mathcal{H}_{G}(M)$ and $\widehat{\mathcal{H}}_{G}(M)$ become $\mathbb{Z}_{2}$-graded algebras. Both $\mathcal{H}_{G}$ and $\widehat{\mathcal{H}}_{G}$ are functorial with respect to maps of $G$-manifolds. In particular the map $M \rightarrow$ pt makes $\mathcal{H}_{G}(M)$ into a module over the ring $\mathcal{H}_{G}(\mathrm{pt})=\left(U(\mathfrak{g})_{\mathbb{C}}\right)^{G}$ of Casimir elements and $\widehat{\mathcal{H}}_{G}(M)$ into a module over the ring $\widehat{\mathcal{H}}_{G}(\mathrm{pt})=\mathcal{E}^{\prime}(G)^{G}$ of invariant distributions on the group.

2.2. Weil models. Below we will need not only the Cartan models of equivariant cohomology but also the equivalent Weil models.

2.2.1. G-differential algebras. The concept of a $G$-differential algebra, introduced by Cartan [2], generalizes the relations between contractions $\iota_{a}$, Lie derivatives $L_{a}$, and exterior differential d on the super-algebra $\Omega^{\star}(M)$ of differential forms on a $G$-manifold.

Define a graded vector-space $\hat{\mathfrak{g}}=\bigoplus_{i \in \mathbb{Z}} \hat{\mathfrak{g}}^{i}$ where $\hat{\mathfrak{g}}^{1}=\mathbb{R}, \hat{\mathfrak{g}}^{-1}=\hat{\mathfrak{g}}^{0}=\mathfrak{g}$ and $\hat{\mathfrak{g}}^{j}=\{0\}$ for $j \neq-1,0,1$. Given $\xi \in \mathfrak{g}$ let $L_{\xi} \in \hat{\mathfrak{g}}^{0}$ and $\iota_{\xi} \in \hat{\mathfrak{g}}^{-1}$ be the corresponding elements, and let $\mathrm{d} \in \hat{\mathfrak{g}}^{1}$ be the generator corresponding to $1 \in \mathbb{R}$. Then $\hat{\mathfrak{g}}$ is a graded Lie algebra with brackets

$$
\begin{gathered}
{\left[\iota_{\xi}, \mathrm{d}\right]=L_{\xi}, \quad\left[L_{\xi}, \iota_{\eta}\right]=\iota_{[\xi, \eta]}, \quad\left[L_{\xi}, L_{\eta}\right]=L_{[\xi, \eta]}} \\
{\left[\iota_{\xi}, \iota_{\eta}\right]=0, \quad\left[L_{\xi}, \mathrm{d}\right]=0, \quad[\mathrm{~d}, \mathrm{~d}]=0}
\end{gathered}
$$

for all $\xi, \eta \in \mathfrak{g}$. Notice that $\hat{\mathfrak{g}}^{0} \cong \mathfrak{g}$ and $\hat{\mathfrak{g}}^{-1} \oplus \hat{\mathfrak{g}}^{0} \cong \mathfrak{g} \rtimes \mathfrak{g}$ are Lie subalgebras both of which are stable under $\mathrm{d}$.

A $G$-differential space is a super-vector space $B$, together with a representation of $\hat{\mathfrak{g}}$ on $B$. The subspace $B^{G}$ annihilated by $\hat{\mathfrak{g}}^{0}$ is called the invariant subspace, the subspace $B_{\text {hor }}$ annihilated by $\hat{\mathfrak{g}}^{-1}$ is called the horizontal subspace, and the subspace $B_{\text {basic }}$ annihilated 
by $\hat{\mathfrak{g}}^{-1} \oplus \hat{\mathfrak{g}}^{0}$ is called the basic subspace. Note that $B_{\text {basic }}$ is preserved under the differential d.

If $B$ carries in addition the structure of a super-algebra 円, and if $\hat{\mathfrak{g}}$ is represented by derivations, then $B$ is called a $G$-differential algebra. If $B_{1}, B_{2}$ are $G$-differential spaces then so is their $\left(\mathbb{Z}_{2}\right.$-graded) tensor product $B_{1} \otimes B_{2}$. A homomorphism of $G$-differential spaces is a homomorphism of super-spaces intertwining the $\hat{\mathfrak{g}}$-representations. Similar definitions hold for $G$-differential algebras.

2.2.2. The Weil algebras $W_{G}$ and $\widehat{W}_{G}$. The super-commutative Weil algebras $W_{G}$ and $\widehat{W}_{G}$ are defined as tensor products

$$
W_{G}=S \mathfrak{g}_{\mathbb{C}}^{*} \otimes \wedge \mathfrak{g}_{\mathbb{C}}^{*}, \quad \widehat{W}_{G}=\mathcal{E}^{\prime}\left(\mathfrak{g}^{*}\right) \otimes \wedge \mathfrak{g}_{\mathbb{C}}^{*},
$$

with $\mathbb{Z}_{2}$-grading inherited from the $\mathbb{Z}_{2}$-grading on the exterior algebra. They carry the structure of $G$-differential algebras, as follows. Let $y^{a} \in \wedge \mathfrak{g}^{*}$ be the generators corresponding to the basis $e^{a}$ of $\mathfrak{g}^{*}$. Let

$$
L_{a}=L_{a} \otimes 1+1 \otimes L_{a}
$$

be the generators for the action on the Weil algebras $W_{G}, \widehat{W}_{G}$ coming from the coadjoint action of $G$ on $\mathfrak{g}^{*}$. The natural contraction operation on $\wedge \mathfrak{g}^{*}$ extends to operators $\iota_{a}=1 \otimes \iota_{a}$. Let the Weil differential $\mathrm{d}^{W}$ be given by the formula

$$
\mathrm{d}^{W}=y^{a}\left(L_{a} \otimes 1\right)+\left(v^{a}-\frac{1}{2} f_{b c}^{a} y^{b} y^{c}\right) \iota_{a} .
$$

Given a $G$-manifold $M$, the horizontal projection $P_{h o r}: \widehat{W}_{G} \rightarrow \mathcal{E}^{\prime}\left(\mathfrak{g}^{*}\right)$ induces isomorphisms between the basic subcomplexes and the Cartan algebras

$$
P_{h o r}^{W}:=P_{h o r} \otimes 1:\left(\widehat{W}_{G} \otimes \Omega(M)\right)_{b a s i c} \rightarrow \widehat{C}_{G}(M) .
$$

As shown in [2] this isomorphism takes $\mathrm{d}=\mathrm{d}^{W} \otimes 1+1 \otimes \mathrm{d}$ to the Cartan differential $\mathrm{d}_{G}$, and it restricts to an ring isomorphism $P_{h o r}^{W}:\left(W_{G} \otimes \Omega(M)\right)_{b a s i c} \cong C_{G}(M)$. Therefore, $H_{G}(M)$ and $\widehat{H}_{G}(M)$ are equivalently the cohomologies of $\left(W_{G} \otimes \Omega(M)\right)_{\text {basic }}$ and $\left(\widehat{W}_{G} \otimes \Omega(M)\right)_{\text {basic }}$, respectively.

2.2.3. Non-commutative Weil algebras. Let the Lie algebra $\mathfrak{g}$ be equipped with an invariant inner product, used to identify $\mathfrak{g} \cong \mathfrak{g}^{*}$, and suppose that the basis $e_{a} \in \mathfrak{g}$ is orthonormal.

Let $\mathrm{Cl}(\mathfrak{g})$ be the Clifford algebra of $\mathfrak{g}$, defined as the quotient of the tensor algebra by the ideal generated by elements of the form $2 \xi \otimes \xi-\xi \cdot \xi$ with $\xi \in \mathfrak{g}$. The generators $x_{a}$ of $\mathrm{Cl}(\mathfrak{g})$ satisfy the super-bracket relations $\left[x_{a}, x_{b}\right]=\delta_{a b}$. The element $\gamma:=-\frac{1}{6} f_{a b c} x_{a} x_{b} x_{c}$ has the beautiful property [17] that its square is a scalar: $\gamma^{2}=-\frac{1}{48} f_{a b c} f_{a b c}$. Hence

$$
\mathrm{d}^{C l}=\operatorname{ad}\left(-\frac{1}{6} f_{a b c} x_{a} x_{b} x_{c}\right): \mathrm{Cl}(\mathfrak{g})_{\mathbb{C}} \rightarrow \mathrm{Cl}(\mathfrak{g})_{\mathbb{C}}
$$

\footnotetext{
${ }^{1}$ In this paper, (super)algebra always means (super)algebra with unit, and a homomorphism between (super)algebras is required to take the unit element to the unit element.
} 
is a differential. Together with contractions $\iota_{a}=\operatorname{ad}\left(x_{a}\right)$ and the Lie derivatives $L_{a}=$ $\operatorname{ad}\left(-\frac{1}{2} f_{a b c} x_{b} x_{c}\right)$ for the adjoint action this makes $\mathrm{Cl}(\mathfrak{g})_{\mathbb{C}}$ into a $G$-differential algebra.

In terms of the identification $\sigma: \mathrm{Cl}(\mathfrak{g}) \rightarrow \wedge \mathfrak{g}$ by the symbol map, $\sigma\left(x_{a_{1}} \ldots x_{a_{k}}\right)=$ $y_{a_{1}} \ldots y_{a_{k}}$ for $a_{1}<\cdots<a_{k}$, the differential $\mathrm{d}^{C l}$ is given by the formula

$$
\mathrm{d}^{C l}=-\frac{1}{2} f_{a b c} y_{b} y_{c} \iota_{a}-\frac{1}{24} f_{a b c} \iota_{a} \iota_{b} \iota_{c} .
$$

The Weil algebras $\mathcal{W}_{G}$ and $\widehat{\mathcal{W}}_{G}$ are the non-commutative $G$-differential algebras

$$
\mathcal{W}_{G}=U(\mathfrak{g})_{\mathbb{C}} \otimes \mathrm{Cl}(\mathfrak{g})_{\mathbb{C}}, \widehat{\mathcal{W}}_{G}=\mathcal{E}^{\prime}(G) \otimes \mathrm{Cl}(\mathfrak{g})_{\mathbb{C}}
$$

with

$$
\begin{aligned}
\iota_{a} & =\operatorname{ad}\left(x_{a}\right) \\
L_{a} & =\operatorname{ad}\left(u_{a}-\frac{1}{2} f_{a b c} x_{b} x_{c}\right) \\
\mathrm{d}^{\mathcal{W}} & =\operatorname{ad}\left(u_{a} x_{a}-\frac{1}{6} f_{a b c} x_{a} x_{b} x_{c}\right) .
\end{aligned}
$$

The operators $L_{a}$ generate the representation of $\mathfrak{g}$ on the Weil algebra induced from the adjoint action on $\mathfrak{g}$.

By [2], Proposition 3.7 we describe the Weil differential in terms of the vector space isomorphism $\widehat{\mathcal{W}}_{G} \cong \mathcal{E}^{\prime}(G) \otimes \wedge \mathfrak{g}$ given by the symbol map:

$$
\mathrm{d}^{\mathcal{W}}=y_{a}\left(L_{a} \otimes 1\right)+\left(\frac{u_{a}^{L}+u_{a}^{R}}{2}-\frac{1}{2} f_{a b c} y_{b} y_{c}\right) \iota_{a}-\frac{1}{24} f_{a b c} \iota_{a} \iota_{b} \iota_{c} .
$$

Given a $G$-manifold $M$, the horizontal projection $P_{\text {hor }}: \widehat{\mathcal{W}}_{G} \rightarrow \mathcal{E}^{\prime}(G)$ induces isomorphisms of differential algebras,

$$
P_{\text {hor }}^{\mathcal{W}}=P_{\text {hor }} \otimes 1:\left(\widehat{\mathcal{W}}_{G} \otimes \Omega(M)\right)_{\text {basic }} \cong \widehat{\mathcal{C}}_{G}(M), \quad\left(\mathcal{W}_{G} \otimes \Omega(M)\right)_{\text {basic }} \cong \mathcal{C}_{G}(M)
$$

so that the equivariant cohomologies $\mathcal{H}_{G}(M)$ and $\widehat{\mathcal{H}}_{G}(M)$ are equivalently the cohomologies of $\left(\mathcal{W}_{G} \otimes \Omega(M)\right)_{\text {basic }}$ and $\left(\widehat{\mathcal{W}}_{G} \otimes \Omega(M)\right)_{\text {basic }}$.

2.2.4. The quantization map. Let $J^{\frac{1}{2}} \in C^{\infty}(\mathfrak{g})^{G}$ be the unique smooth square root of the determinant of the Jacobian of the exponential map such that $J^{\frac{1}{2}}(0)=1$. The Duflo map [10] is the linear map

$$
\text { Duf : } \mathcal{E}^{\prime}(\mathfrak{g}) \rightarrow \mathcal{E}^{\prime}(G), \quad v \mapsto \exp _{*}\left(J^{\frac{1}{2}} v\right)
$$

It takes distributions supported at the origin to distributions supported at the group unit, hence defines a vector space isomorphism Duf $: S(\mathfrak{g}) \rightarrow U(\mathfrak{g})$. The important property of the Duflo map is that it becomes a ring homomorphism if restricted to invariant elements.

In [2] we define an explicit homomorphism of $G$-differential spaces, called the quantization map,

$$
\mathcal{Q}: \widehat{W}_{G} \rightarrow \widehat{\mathcal{W}}_{G}
$$


which on $W_{G}$ restricts to an isomorphism, $W_{G} \rightarrow \mathcal{W}_{G}$. On $1 \otimes \wedge \mathfrak{g}_{\mathbb{C}}^{*}$ the map $\mathcal{Q}$ coincides with the symbol map, and on $\mathcal{E}^{\prime}(\mathfrak{g}) \otimes 1$ it is equal to the Duflo map. Moreover, we show that the induced homomorphisms in cohomology preserve the ring structures, i.e. the map $\widehat{H}_{G}(M) \rightarrow \widehat{\mathcal{H}}_{G}(M)$ is an algebra homomorphism and the map $H_{G}(M) \rightarrow \mathcal{H}_{G}(M)$ is an algebra isomorphism.

\section{Abelianization}

The equivariant cohomology groups $H_{G}(M)$ and $\widehat{H}_{G}(M)$ are functorial not only with respect to maps of $G$-manifolds but also with respect to group homomorphisms. In particular, the inclusion of the maximal torus $T$ induces maps $R_{G}^{T}: H_{G}(M) \rightarrow H_{T}(M)$ and $R_{G}^{T}: \widehat{H}_{G}(M) \rightarrow \widehat{H}_{T}(M)$. Both of these maps are injective, and their image is the subalgebra of Weyl invariants.

This functoriality property does not carry over to group-valued equivariant cohomology $\widehat{\mathcal{H}}_{G}(M)$, because a group homomorphism $\phi: K \rightarrow G$ does not give rise to $K$-equivariant maps $\mathcal{W}_{G} \rightarrow \mathcal{W}_{K}$ or $\widehat{\mathcal{W}}_{G} \rightarrow \widehat{\mathcal{W}}_{K}$ in any natural way.

It is the goal of this section to obtain a suitable replacement for the restriction map $R_{G}^{T}$. The main steps are as follows.

a. First, we define a new Weil algebra $\widehat{\mathcal{W}}_{T}^{-}=\mathcal{E}^{\prime}(T)^{-} \otimes \mathrm{Cl}(\mathfrak{t})_{\mathbb{C}}$, involving the negative Hardy space $\mathcal{E}^{\prime}(T)^{-}$.

b. We construct a map $r_{G}^{T}: \mathcal{E}^{\prime}(G) \rightarrow \mathcal{E}^{\prime}(T)^{-}$with nice properties.

c. We define a map $p_{G}^{T} \circ \mathcal{T}: \mathrm{Cl}(\mathfrak{g})_{\mathbb{C}} \rightarrow \mathrm{Cl}(\mathfrak{t})_{\mathbb{C}}$, where $p_{G}^{T}$ is orthogonal projection and the "twist" operator $\mathcal{T}$ involves the classical r-matrix. We show that this map intertwines the Clifford differentials "up to a $\rho$-shift".

d. The restriction map $\mathcal{R}_{G}^{T}: \widehat{\mathcal{W}}_{G} \rightarrow \widehat{\mathcal{W}}_{T}^{-}$will be defined as a product of the two maps, $r_{G}^{T}$ and $p_{G}^{T} \circ \mathcal{T}$. The main result of this Section is Theorem 3.7, stating that $\mathcal{R}_{G}^{T}$ is a homomorphism of $T$-differential spaces. It therefore defines a map in equivariant cohomology.

Throughout this section we will assume that $G$ is a product of a simply connected group and a torus (although only the simply connected part will play a role). A given invariant inner product on $\mathfrak{g}$ will be used to identify $\mathfrak{g} \cong \mathfrak{g}^{*}$.

We let $T$ be a maximal torus of $G$, with Lie algebra $\mathfrak{t}$, and denote by $\mathfrak{t}_{+}$some choice of a fundamental Weyl chamber. Let $\Lambda \subset \mathfrak{t}$ be the integral lattice, its dual $\Lambda^{*} \subset \mathfrak{t}$ the weight lattice, and $\Lambda_{+}^{*}=\Lambda^{*} \cap \mathfrak{t}_{+}$the set of dominant weights.

Given $t \in T$ and $\lambda \in \Lambda^{*}$ we denote by $t^{\lambda} \in S^{1} \subset \mathbb{C}$ the image of $t$ under the $T$-character $T \rightarrow S^{1}$ defined by $\lambda$, that is $(\exp \xi)^{\lambda}=e^{2 \pi i\langle\lambda, \xi\rangle}$. Abusing notation we sometimes denote the map itself by $t^{\lambda}$.

Let $\mathfrak{R} \subset \Lambda^{*}$ be the set of roots of $G$ and $\mathfrak{R}_{+}$the subset of positive roots. Our assumption that $G$ be a product of a connected, simply connected Lie group and a 
torus, implies that the half-sum of positive roots

$$
\rho=\frac{1}{2} \sum_{\alpha \in \Re_{+}} \alpha
$$

is a weight $\rho \in \Lambda_{+}^{*}$. It is alternatively characterized as the sum of fundamental weights for the semi-simple part of $G$.

For every positive root $\alpha \in \mathfrak{R}_{+}$let $e_{\alpha} \in \mathfrak{g}_{\mathbb{C}}$ be a root vector, with property $\left[\xi, e_{\alpha}\right]=$ $2 \pi i\langle\alpha, \xi\rangle e_{\alpha}$ for $\xi \in \mathfrak{t}$, and set $e_{-\alpha}=\overline{e_{\alpha}}$. Normalize the length of $e_{\alpha}$ so that $e_{\alpha} \cdot e_{-\alpha}=1$. Then

$$
\left[e_{\alpha}, e_{-\alpha}\right]=2 \pi i \alpha .
$$

Every weight $\lambda \in \Lambda_{+}^{*}$ parametrizes a unique irreducible $G$-representation $V_{\lambda}$, containing a highest weight vector $v_{\lambda} \neq 0$, with

$$
e_{\alpha} \cdot v_{\lambda}=0
$$

for every positive root $\alpha \in \mathfrak{R}_{+}$. and

$$
t \cdot v_{\lambda}=t^{\lambda} v_{\lambda}
$$

for every $t \in T$. We denote by $\chi_{\lambda}$ the character of the representation $V_{\lambda}$.

3.1. The Hardy-Weil algebra $\widehat{\mathcal{W}}_{T}^{-}$. Recall (cf. [11], Chapter 8.5) that for any distribution $u \in \mathcal{E}^{\prime}(T)$, the Fourier coefficients $\left\langle u, t^{\lambda}\right\rangle$ are polynomially bounded:

$$
\left|\left\langle u, t^{\lambda}\right\rangle\right| \leq C(1+|\lambda|)^{N}
$$

for some $C>0, N \in \mathbb{Z}$. Conversely every sequence of complex numbers satisfying such an estimate defines a distribution on $T$. We can therefore identify $\mathcal{E}^{\prime}(T)$ with the space of polynomially bounded sequences $\Lambda^{*} \rightarrow \mathbb{C}$. Similarly, if $\phi \in C^{\infty}(T)$ has Fourier decomposition $\phi=\sum_{\nu \in \Lambda^{*}} a_{\nu} t^{\nu}$ then the coefficients $a_{\nu}$ are rapidly decreasing, that is for all $k>0$ there exists $C_{k}$ with

$$
\left|a_{\nu}\right| \leq C_{k}(1+|\nu|)^{-k}
$$

Conversely, every rapidly decreasing sequence $a_{\nu}$ defines a smooth function on the torus.

Let the negative Hardy space $\mathcal{E}^{\prime}(T)^{-} \subset \mathcal{E}^{\prime}(T)$ consist of all distributions on $T$ for which only strictly negative Fourier coefficients are non-vanishing,

$$
\mathcal{E}^{\prime}(T)^{-}:=\left\{u \in \mathcal{E}^{\prime}(T) \mid\left\langle u, t^{\nu}\right\rangle=0 \text { if } \nu \notin\left(\rho+\Lambda_{+}^{*}\right)\right\},
$$

and let $\pi^{-}$be the Szegö projector onto this space,

$$
\left\langle\pi^{-}(u), t^{\lambda+\rho}\right\rangle=\left\{\begin{array}{cl}
\left\langle u, t^{\nu}\right\rangle & \text { if } \lambda \in \Lambda_{+}^{*} \\
0 & \text { otherwise }
\end{array}\right.
$$

Convolution of distributions makes the Hardy space into an algebra with unit element $\delta_{e}^{-}:=\pi^{-}\left(\delta_{e}\right)$. The Szegö projector is an algebra homomorphism. (The inclusion of $\mathcal{E}^{\prime}(T)^{-}$into $\mathcal{E}^{\prime}(T)$ is not an algebra homomorphism since the unit elements are different.) 
The Hardy-Weil algebra is defined as the tensor product

$$
\widehat{\mathcal{W}}_{T}^{-}:=\mathcal{E}^{\prime}(T)^{-} \otimes \mathrm{Cl}(\mathfrak{t})_{\mathbb{C}} .
$$

It is a $T$-differential algebra, with differential $\mathrm{d}^{-}:=\sum_{k=1}^{r} u_{k}^{-} \iota_{k}$ where $u_{k}^{-}=\pi^{-}\left(u_{k}\right)$. The projection $\pi^{-}:=\pi^{-} \otimes 1: \widehat{\mathcal{W}}_{T} \rightarrow \widehat{\mathcal{W}}_{T}^{-}$is a homomorphism of $T$-differential algebras and the inclusion $\widehat{\mathcal{W}}_{T}^{-} \hookrightarrow \widehat{W}_{T}$ is a homomorphism of $T$-differential spaces (not algebras).

Given a $T$-manifold $M$, let $\widehat{\mathcal{H}}_{T}^{-}(M)$ be the cohomology of the basic subcomplex $\left(\widehat{\mathcal{W}}_{T}^{-} \otimes\right.$ $\Omega(M))_{\text {basic }}$. Equivalently, it is the cohomology for the Cartan model,

$$
\widehat{\mathcal{C}}_{T}^{-}(M)=\mathcal{E}^{\prime}(T)^{-} \otimes \Omega(M)^{T}, \quad \mathrm{~d}_{T}^{-}:=1 \otimes \mathrm{d}-\sum_{k=1}^{r} u_{k}^{-} \otimes \iota_{k} .
$$

The projection $\widehat{\mathcal{W}}_{T} \rightarrow \widehat{\mathcal{W}}_{T}^{-}$gives rise to a ring homomorphism $\widehat{\mathcal{H}}_{T}(M) \rightarrow \widehat{\mathcal{H}}_{T}^{-}(M)$, and the inclusion $\widehat{\mathcal{W}}_{T}^{-} \hookrightarrow \widehat{W}_{T}$ defines a linear map $\widehat{\mathcal{H}}_{T}^{-}(M) \rightarrow \widehat{\mathcal{H}}_{T}(M)$.

3.2. The restriction map $r_{G}^{T}: \mathcal{E}^{\prime}(G) \rightarrow \mathcal{E}^{\prime}(T)^{-}$. Below we will often find it convenient to work with a Cartan-Weyl basis in $\mathfrak{g}_{\mathbb{C}}$, given by the collection of $e_{\alpha}, e_{-\alpha}$ supplemented by a basis $e_{k}(k=1, \ldots, r)$ for $\mathfrak{t}$.

For all $\lambda \in \Lambda_{+}^{*}$ let $v_{\lambda}$ be a highest weight vector of unit length, and define a function $\Delta_{\lambda} \in C^{\infty}(G)$ as the matrix element

$$
\Delta_{\lambda}(g)=\left(v_{\lambda}, g \cdot v_{\lambda}\right),
$$

using the inner product $(\cdot, \cdot)$ on $V_{\lambda}$. It has the properties

$$
\begin{gathered}
\Delta_{\lambda}(e)=1, \\
\Delta_{\lambda}(t g)=\Delta_{\lambda}(g t)=t^{\lambda} \Delta_{\lambda}(g), \quad(t \in T, g \in G), \\
e_{\alpha}^{L} \Delta_{\lambda}=e_{-\alpha}^{R} \Delta_{\lambda}=0 .
\end{gathered}
$$

for $\alpha \in \mathfrak{R}_{+}$, where for any $\xi \in \mathfrak{g}_{\mathbb{C}}$ we denote by $\xi^{L}, \xi^{R}$ the left-and right invariant vector fields equal to $\xi$ at the group unit. In fact, because of the decomposition

$$
L^{2}(G)=\bigoplus_{\lambda \in \Lambda_{+}^{*}} V_{\lambda} \otimes V_{\lambda}^{*}=\bigoplus_{\lambda \in \Lambda_{+}^{*}} \operatorname{End}\left(V_{\lambda}\right)
$$

as a $G \times G$ representation, the function $\Delta_{\lambda}$ is uniquely characterized by these three properties. The functions $\Delta_{\lambda}$ have the property

$$
\Delta_{\lambda+\nu}=\Delta_{\lambda} \Delta_{\nu}
$$

for all $\lambda, \nu \in \Lambda_{+}^{*}$.

Example 3.1. Let $G=S U(2)$. All dominant weights are multiples $\lambda=k \rho$ with $k=$ $0,1, \ldots$, and

$$
\Delta_{k \rho}\left(\begin{array}{ll}
a & b \\
c & d
\end{array}\right)=a^{k} .
$$


Proposition 3.2. The $\operatorname{map} r_{G}^{T}: \mathcal{E}^{\prime}(G) \rightarrow \mathcal{E}^{\prime}(T)^{-}$,

$$
\left\langle r_{G}^{T}(u), t^{\lambda+\rho}\right\rangle=\left\langle u, \Delta_{\lambda}\right\rangle
$$

is well-defined and continuous.

Proof. To show that $\left\langle u, \Delta_{\lambda}\right\rangle$ are Fourier coefficients for a well-defined distribution $r_{G}^{T}(u) \in$ $\mathcal{E}^{\prime}(T)^{-}$, we have to show that they are polynomially bounded as a function of $\lambda$. Clearly, this only involves the semi-simple part of $G$ (indeed, if $G$ is abelian, there is nothing to prove since $\mathcal{E}^{\prime}(T)^{-}=\mathcal{E}^{\prime}(G)$ in that case and the map $r_{G}^{T}$ is the identity map).

Assume therefore that $G$ is simply connected. Then $\Lambda_{+}^{*}$ is spanned by fundamental weights $w_{1}, \ldots, w_{r}$ of $G$. Let

$$
\Delta:=\left(\Delta_{w_{1}}, \ldots, \Delta_{w_{r}}\right): G \rightarrow \mathbb{C}^{r} .
$$

By (13), $\Delta_{\lambda}=\Delta^{*} z^{\lambda}$ using multi-index notation $z^{\lambda}=\prod_{j} z_{j}^{\lambda_{j}}$, hence $\left\langle u, \Delta_{\lambda}\right\rangle=\left\langle\Delta_{*} u, z^{\lambda}\right\rangle$. The image of $\Delta$ is contained in the unit polydisk $D=\left\{\left(z_{1}, \ldots, z_{r}\right) \in \mathbb{C}^{r}|| z_{k} \mid \leq 1\right\} \subset \mathbb{C}^{r}$.

For any distribution $v \in \mathcal{E}^{\prime}\left(\mathbb{C}^{r}\right)$ supported on the polydisk $D$, there exist constants $C>0$ and $k \in \mathbb{N}$ such that the value of $v$ on any test function $\phi$ is bounded by derivatives of $\phi$ up to order $k$ (cf. Hörmander [13], Theorem 2.3.10)

$$
|(v, \phi)| \leq C \sum_{|J| \leq k} \sup _{x \in D}\left|\frac{\partial^{|J|} \phi}{\partial x^{J}}\right| .
$$

Applying this to $v=\Delta_{*} u$ and $\phi=z^{\lambda}$ immediately gives the required estimates.

To show that $r_{G}^{T}$ is continuous, consider the semi-norms $\left\langle r_{G}^{T}(u), \phi\right\rangle$ for $\phi \in C^{\infty}(T)$. Since the coefficients $a_{\nu}$ in the Fourier decomposition

$$
\phi=\sum_{\nu \in \Lambda^{*}} a_{\nu} t^{\nu} \in C^{\infty}(T)
$$

are rapidly decreasing, and since $\left|\Delta_{\lambda}\right| \leq 1$, the function

$$
\psi:=\sum_{\lambda \in \Lambda_{+}^{*}} a_{\lambda+\rho} \Delta_{\lambda}
$$

on $G$ is well-defined and continuous. Let $B$ be the Laplace operator on $G$. On $V_{\lambda} \otimes V_{\lambda}^{*} \subset$ $L^{2}(G)$ it acts as a scalar $4 \pi^{2}\left(|\lambda+\rho|^{2}-|\rho|^{2}\right)$. Hence

$$
B^{k} \psi=\sum_{\lambda \in \Lambda_{+}^{*}}\left(4 \pi^{2}\left(|\lambda+\rho|^{2}-|\rho|^{2}\right)\right)^{k} a_{\lambda+\rho} \Delta_{\lambda}
$$

is still continuous. By the Sobolev Lemma, this shows that $\psi$ is smooth. Since $\left\langle r_{G}^{T}(u), \phi\right\rangle=$ $\langle u, \psi\rangle$ it follows that $r_{G}^{T}$ is continuous.

Example 3.3. Given $h \in T$, let $\delta_{h}^{-} \in \mathcal{E}^{\prime}(T)^{-}$be the distribution

$$
\delta_{h}^{-}=\pi^{-}\left(\delta_{h}^{T}\right),
$$


where $\delta_{h}^{T}$ is the delta-distribution on $T$. Note that

$$
\delta_{h_{1}}^{-} \delta_{h_{2}}^{-}=\delta_{h_{1} h_{2}}^{-}
$$

for $h_{1}, h_{2} \in T$. The distribution $\delta_{h}^{-}$on $T$ is related to the delta-distribution $\delta_{h}$ on $G$ by

$$
h^{\rho} r_{G}^{T}\left(\delta_{h}\right)=\delta_{h}^{-},
$$

by the calculation $\left\langle r_{G}^{T}\left(\delta_{h}\right), t^{\lambda+\rho}\right\rangle=\Delta_{\lambda}(h)=h^{\lambda}$.

Proposition 3.4 (Properties of the map $\left.r_{G}^{T}\right)$. Let $u_{-\alpha}, u_{k}, u_{\alpha}$ be the generators of $U(\mathfrak{g})_{\mathbb{C}}$ in the Cartan-Weyl basis. Then

$$
\begin{aligned}
r_{G}^{T} \circ\left(u_{k}\right)^{R} & =r_{G}^{T} \circ\left(u_{k}\right)^{L}=\left(u_{k}^{-}-2 \pi i \rho_{k}\right) \circ r_{G}^{T} \\
r_{G}^{T} \circ\left(u_{-\alpha}\right)^{L} & =r_{G}^{T} \circ\left(u_{\alpha}\right)^{R}=0
\end{aligned}
$$

for $k=1, \ldots, r$ and all $\alpha \in \mathfrak{R}_{+}$. Here the superscripts $L / R$ denote multiplication from the left/right.

Proof. Let $u \in \mathcal{E}^{\prime}(G)$ and $\lambda \in \Lambda_{+}^{*}$ be given. Then

$$
\left\langle u_{k}^{-} \circ r_{G}^{T}(u), t^{\lambda+\rho}\right\rangle=2 \pi i\left(\lambda_{k}+\rho_{k}\right)\left\langle r_{G}^{T}(u), t^{\lambda+\rho}\right\rangle
$$

while on the other hand

$$
\left\langle r_{G}^{T}\left(u_{k} u\right), t^{\lambda+\rho}\right\rangle=\left\langle\left(u_{k} u\right), \Delta_{\lambda}\right\rangle=\left\langle u, e_{k}^{R} \Delta_{\lambda}\right\rangle=2 \pi i \lambda_{k}\left\langle u, \Delta_{\lambda}\right\rangle=2 \pi i \lambda_{k}\left\langle r_{G}^{T}(u), t^{\lambda+\rho}\right\rangle .
$$

Hence, $r_{G}^{T} \circ\left(u_{k}\right)^{L}=\left(u_{k}^{-}-2 \pi i \rho_{k}\right) \circ r_{G}^{T}$. Similarly, $r_{G}^{T} \circ\left(u_{k}\right)^{R}=\left(u_{k}^{-}-2 \pi i \rho_{k}\right) \circ r_{G}^{T}$, proving (14). The equations (15) are obtained from (12) as follows:

$$
\begin{gathered}
\left\langle r_{G}^{T}\left(u_{-\alpha} u\right), t^{\lambda+\rho}\right\rangle=\left\langle u_{-\alpha} u, \Delta_{\lambda}\right\rangle=-\left\langle u, e_{-\alpha}^{R} \Delta_{\lambda}\right\rangle=0, \\
\left\langle r_{G}^{T}\left(u u_{\alpha}\right), t^{\lambda+\rho}\right\rangle=\left\langle u u_{\alpha}, \Delta_{\lambda}\right\rangle=\left\langle u, e_{\alpha}^{L} \Delta_{\lambda}\right\rangle=0 .
\end{gathered}
$$

The function $\Delta_{\lambda}$ is $\operatorname{Ad}(T)$-invariant but not $\operatorname{Ad}(G)$-invariant. Let $P^{G}: C^{\infty}(G) \rightarrow$ $C^{\infty}(G)^{G}$ be projection to the $\operatorname{Ad}(G)$-invariant part (given by averaging over the group). We claim that

$$
P^{G}\left(\Delta_{\lambda}\right)=\left(\operatorname{dim} V_{\lambda}\right)^{-1} \chi_{\lambda} .
$$

To see this let $\tau_{\lambda}: G \rightarrow \operatorname{Aut}\left(V_{\lambda}\right)$ be the representation labeled by $\lambda$. For any weight $\nu \in \Lambda_{+}^{*}$, the operator $\int_{G} \tau_{\lambda}(g) \overline{\chi_{\nu}(g)} \mathrm{d} g$ commutes with all $\tau_{\lambda}(h)$ and is therefore a multiple of the identity:

$$
\int_{G} \tau_{\lambda}(g) \overline{\chi_{\nu}(g)} \mathrm{d} g=\delta_{\nu, \lambda} \frac{\operatorname{Vol} G}{\operatorname{dim} V_{\lambda}} \operatorname{Id}_{V_{\lambda}},
$$

where the constant is verified by taking the trace of both sides. As a consequence,

$$
\int_{G} \Delta_{\lambda}(g) \overline{\chi_{\nu}(g)} \mathrm{d} g=\int_{G}\left(v_{\lambda}, g \cdot v_{\lambda}\right) \overline{\chi_{\nu}(g)} \mathrm{d} g=\delta_{\nu, \lambda} \frac{\operatorname{Vol} G}{\operatorname{dim} V_{\lambda}}
$$

which implies (16). 
3.3. Restriction for $\mathrm{Cl}(\mathfrak{g})_{\mathbb{C}}$. As our next ingredient in the definition of the map $\mathcal{R}_{G}^{T}$ : $\widehat{\mathcal{W}}_{G} \rightarrow \widehat{\mathcal{W}}_{T}^{-}$we need a suitable projection from $\mathrm{Cl}(\mathfrak{g})_{\mathbb{C}} \rightarrow \mathrm{Cl}(\mathfrak{t})_{\mathbb{C}}$. Let $\mathrm{Cl}(\mathfrak{g})_{\mathbb{C}}=\mathrm{Cl}(\mathfrak{t})_{\mathbb{C}} \otimes$ $\mathrm{Cl}\left(\mathfrak{t}^{\perp}\right)_{\mathbb{C}}$ be the decomposition of the Clifford algebra, and $p_{G}^{T}: \mathrm{Cl}(\mathfrak{g})_{\mathbb{C}} \rightarrow \mathrm{Cl}(\mathfrak{t})_{\mathbb{C}}$ the orthogonal projection defined by it. We will pre-compose this projection with a "twist"

$$
\mathcal{T}=\exp \left(\frac{1}{2} r_{a b} \iota_{a} \iota_{b}\right)
$$

where $r=r_{a b} e_{a} e_{b} \in \wedge^{2} \mathfrak{g}_{\mathbb{C}}$ is the classical r-matrix,

$$
r=\sum_{\alpha} e_{\alpha} \wedge e_{-\alpha}=\frac{1}{2} \sum_{\alpha}\left(e_{\alpha} \otimes e_{-\alpha}-e_{-\alpha} \otimes e_{\alpha}\right),
$$

Recall the following properties of the r-matrix:

Proposition 3.5 (Properties of the classical r-matrix). The r-matrix $r \in \wedge^{2} \mathfrak{g}_{\mathbb{C}}$ satisfies the equations

a. (Classical Yang-Baxter Equation)

$$
\mathrm{Cycl}_{a b c}\left(r_{a s} f_{s b t} r_{t c}\right)=\frac{1}{4} f_{a b c}
$$

where $\mathrm{Cycl}_{a b c}$ is the sum over cyclic permutations. b.

$$
f_{a b c} r_{b c}=4 \pi i \rho_{a}
$$

c. For any $\mu \in \mathfrak{t}$,

$$
\mu_{a}\left(f_{a b s} r_{s c}-f_{a s c} r_{b s}\right)=0 .
$$

We include the proof of Proposition 3.5, since many different normalizations of the $r$-matrix appear in the literature.

Proof. Using the Cartan-Weyl basis for $\mathfrak{g}_{\mathbb{C}}$, the only non-vanishing entries in $r_{a b}$ are $r_{-\alpha, \alpha}=-r_{\alpha,-\alpha}=1 / 2$. Up to permutation of indices, the only non-vanishing structure constants are of the form $f_{-\alpha, \alpha, i}, f_{-\alpha-\beta, \alpha, \beta}$. One verifies:

$$
\begin{aligned}
r_{-\alpha, \alpha} f_{-\alpha, i, \alpha} r_{-\alpha, \alpha} & =\frac{1}{4} f_{-\alpha, i, \alpha}, \\
r_{-\alpha, \alpha} f_{-\alpha,-\beta, \alpha+\beta} r_{-\alpha-\beta, \alpha+\beta}+r_{-\beta, \beta} f_{-\beta, \alpha+\beta,-\alpha} r_{\alpha,-\alpha} & \\
+r_{\alpha+\beta,-\alpha-\beta} f_{\alpha+\beta,-\alpha,-\beta} r_{\beta,-\beta} & =\frac{1}{4} f_{-\alpha,-\beta, \alpha+\beta} .
\end{aligned}
$$

Equation (18) follows from the calculation, using (6),

$$
r_{a b} f_{a b c} e_{c}=\sum_{\alpha \in \mathfrak{R}_{+}}\left[e_{\alpha}, e_{-\alpha}\right]=2 \pi i \sum_{\alpha \in \mathfrak{R}_{+}} \alpha=4 \pi i \rho .
$$

The last Equation (19) is just the infinitesimal version of the $T$-invariance of $r$.

The main property of the composition $p_{G}^{T} \circ \mathcal{T}$ is that it intertwines the Clifford differential on $\mathrm{Cl}(\mathfrak{g})_{\mathbb{C}}$ with a very simple differential on $\mathrm{Cl}(\mathfrak{t})_{\mathbb{C}}$ : 
Proposition 3.6. The composition $p_{G}^{T} \circ \mathcal{T}$ intertwines the Clifford differential $d^{C l}$ on $\mathrm{Cl}(\mathfrak{g})_{\mathbb{C}}$ and the differential $2 \pi i \sum_{k=1}^{r} \rho_{k} \iota_{k}$ on $\mathrm{Cl}(\mathfrak{t})_{\mathbb{C}}$ :

$$
\left(p_{G}^{T} \circ \mathcal{T}\right) \circ d^{C l}=\left(2 \pi i \sum_{k=1}^{r} \rho_{k} \iota_{k}\right) \circ\left(p_{G}^{T} \circ \mathcal{T}\right) .
$$

Proof. We use the symbol map $\sigma: \mathrm{Cl}(\mathfrak{g}) \rightarrow \wedge \mathfrak{g}$ to identify $\mathrm{d}^{C l}$ with the differential (3) on $\wedge \mathfrak{g}_{\mathbb{C}}$. Since $\operatorname{Ad}(\mathcal{T}) y_{a}=y_{a}-r_{a b} \iota_{b}$, we find

$$
\operatorname{Ad}(\mathcal{T}) \mathrm{d}^{C l}=\left(-\frac{1}{2} f_{a b c}\left(y_{b}-r_{b l} \iota_{l}\right)\left(y_{c}-r_{c m} \iota_{m}\right)\right) \iota_{a}-\frac{1}{24} f_{a b c} \iota_{a} \iota_{b} \iota_{c} .
$$

In this expression, the terms cubic in contractions $\iota_{a}$ cancel thanks to the Yang-Baxter equation (17) for $r$. The remaining terms combine, using (18), to

$$
\begin{aligned}
\operatorname{Ad}(\mathcal{T}) \mathrm{d}^{C l} & =-\frac{1}{2} f_{a b c} y_{b} y_{c} \iota_{a}+\frac{1}{2} f_{a b c}\left(r_{c m} y_{b} \iota_{m} \iota_{a}+r_{b l} \iota_{l} y_{c} \iota_{a}\right) \\
& =-\frac{1}{2} f_{a b c} y_{b} y_{c} \iota_{a}+f_{a b c}\left(r_{c m} y_{b} \iota_{m} \iota_{a}+\frac{1}{2} r_{b c} \iota_{a}\right) \\
& =-\frac{1}{2} f_{a b c} y_{b} y_{c} \iota_{a}+f_{a b d} r_{d c} y_{a} \iota_{b} \iota_{c}+2 \pi i \rho_{a} \iota_{a} .
\end{aligned}
$$

Composing this operator with $p_{G}^{T}$ kills the first term, and also the second since

$$
p_{G}^{T} \circ\left(y_{a} f_{a b d} r_{d c} \iota_{b} \iota_{c}\right)=\sum_{k=1}^{r} y_{k} \circ p_{G}^{T} \circ f_{k b d} r_{d c} \iota_{b} \iota_{c}=0
$$

where we have used Equation (19). We conclude

$$
p_{G}^{T} \circ \operatorname{Ad}(\mathcal{T}) \mathrm{d}^{C l}=2 \pi i p_{G}^{T} \circ \sum_{k=1}^{r} \rho_{k} \iota_{k}=2 \pi i \sum_{k=1}^{r} \rho_{k} \iota_{k} \circ p_{G}^{T} .
$$

3.4. Restriction for $\widehat{\mathcal{W}}_{G}$. Taking the product of the restriction maps $r_{G}^{T}: \mathcal{E}^{\prime}(G) \rightarrow$ $\mathcal{E}^{\prime}(T)^{-}$and $\left(p_{G}^{T} \circ \mathcal{T}\right): \mathrm{Cl}(\mathfrak{g})_{\mathbb{C}} \rightarrow \mathrm{Cl}(\mathfrak{t})_{\mathbb{C}}$ we obtain a map between Weil algebras,

$$
\mathcal{R}_{G}^{T}:=r_{G}^{T} \otimes\left(p_{G}^{T} \circ \mathcal{T}\right): \widehat{\mathcal{W}}_{G} \rightarrow \widehat{\mathcal{W}}_{T}^{-} .
$$

The main result of this Section reads:

Theorem 3.7. The map $\mathcal{R}_{G}^{T}: \widehat{\mathcal{W}}_{G} \rightarrow \widehat{\mathcal{W}}_{T}^{-}$is a homomorphism of T-differential spaces. That is, it is a chain map which moreover intertwines the contractions and Lie derivatives for the T-action.

Proof. It is clear that $\mathcal{R}_{G}^{T}$ commutes with Lie derivatives $L_{\xi}$ and contractions $\iota_{\xi}$ for all $\xi \in$ t. To show that $\mathcal{R}_{G}^{T}$ is a chain map we show that $\left(r_{G}^{T} \otimes p_{G}^{T}\right) \circ \mathrm{Ad}(\mathcal{T}) \mathrm{d}^{\mathcal{W}}=\mathrm{d}^{\mathcal{W}} \circ\left(r_{G}^{T} \otimes p_{G}^{T}\right)$. By definition, the Weil differential splits into two pieces $\mathrm{d}^{\mathcal{W}}=\mathrm{d}^{\prime}+\mathrm{d}^{C l}$, with $\mathrm{d}^{\prime}=\operatorname{ad}\left(u_{a} x_{a}\right)$. 
Again we identify $\mathrm{Cl}(\mathfrak{g}) \cong \wedge \mathfrak{g}$ by the symbol map. Since $x_{a}^{L}=y_{a}-\frac{1}{2} \iota_{a}$ and $x_{a}^{R}=y_{a}+\frac{1}{2} \iota_{a}$, we have:

$$
\mathrm{d}^{\prime}=y_{a}\left(u_{a}^{L}-u_{a}^{R}\right)+\frac{1}{2}\left(u_{a}^{L}+u_{a}^{R}\right) \iota_{a} .
$$

Conjugating by $\mathcal{T}$, and using $\operatorname{Ad}(\mathcal{T}) y_{a}=y_{a}-r_{a b} \iota_{b}$,

$$
\operatorname{Ad}(\mathcal{T}) \mathrm{d}^{\prime}=\left(y_{a}-r_{a b} \iota_{b}\right)\left(u_{a}^{L}-u_{a}^{R}\right)+\frac{1}{2}\left(u_{a}^{L}+u_{a}^{R}\right) \iota_{a} .
$$

In the Cartan-Weyl basis,

$$
r_{a b} \iota_{b}\left(u_{a}^{L}-u_{a}^{R}\right)=\frac{1}{2} \sum_{\alpha \in \Re_{+}}\left(\left(u_{\alpha}^{L}-u_{\alpha}^{R}\right) \iota_{-\alpha}-\left(u_{-\alpha}^{L}-u_{-\alpha}^{R}\right) \iota_{\alpha}\right)
$$

while

$$
\frac{1}{2}\left(u_{a}^{L}+u_{a}^{R}\right) \iota_{a}=\frac{1}{2} \sum_{k=1}^{r}\left(u_{k}^{L}+u_{k}^{R}\right) \iota_{k}+\frac{1}{2} \sum_{\alpha \in \Re_{+}}\left(\left(u_{\alpha}^{L}+u_{\alpha}^{R}\right) \iota_{-\alpha}+\left(u_{-\alpha}^{L}+u_{-\alpha}^{R}\right) \iota_{\alpha}\right) .
$$

Hence,

$$
\operatorname{Ad}(\mathcal{T}) \mathrm{d}^{\prime}=L_{a} \otimes y_{a}+\sum_{\alpha \in \Re_{+}}\left(u_{-\alpha}^{L} \iota_{\alpha}+u_{\alpha}^{R} \iota_{-\alpha}\right)+\sum_{k=1}^{r}\left(\frac{u_{k}^{L}+u_{k}^{R}}{2}\right) \iota_{k} .
$$

Next, apply $r_{G}^{T} \otimes p_{G}^{T}$ to this expression. This kills the first term since

$$
\left(r_{G}^{T} \otimes p_{G}^{T}\right)\left(L_{a} \otimes y_{a}\right)=\sum_{k=1}^{r}\left(r_{G}^{T} \circ L_{k}\right) \otimes y_{k}=0
$$

(by $\operatorname{Ad}(T)$-invariance of $r_{G}^{T}$ ). Since $r_{G}^{T} \circ u_{-\alpha}^{L}=r_{G}^{T} \circ u_{\alpha}^{R}=0$, the second term is killed as well. Only the last term survives and using (14) we find

$$
\begin{aligned}
\left(r_{G}^{T} \otimes p_{G}^{T}\right) \circ \operatorname{Ad}(\mathcal{T}) \mathrm{d}^{\prime} & =\left(r_{G}^{T} \otimes p_{G}^{T}\right) \sum_{k=1}^{r}\left(\frac{u_{k}^{L}+u_{k}^{R}}{2}\right) \iota_{k} \\
& =\sum_{k=1}^{r}\left(u_{k}^{-}-2 \pi i \rho_{k}\right) \iota_{k} \circ\left(r_{G}^{T} \otimes p_{G}^{T}\right) .
\end{aligned}
$$

Together with Proposition 3.6 this shows

$$
\left(r_{G}^{T} \otimes p_{G}^{T}\right) \circ \operatorname{Ad}(\mathcal{T})\left(\mathrm{d}^{\prime}+d^{C l}\right)=\sum_{k=1}^{r} u_{k}^{-} \iota_{k} \circ\left(r_{G}^{T} \otimes p_{G}^{T}\right)
$$

which completes the proof. 
3.5. Restriction $\mathcal{R}_{G}^{T}$ in cohomology. For every $G$-manifold $M$, the homomorphism of $T$-differential algebras $\mathcal{R}_{G}^{T}: \widehat{\mathcal{W}}_{G} \rightarrow \widehat{\mathcal{W}}_{T}^{-}$studied in the previous section induces a chain map between the basic subcomplexes

$$
\mathcal{R}_{G}^{T}=\mathcal{R}_{G}^{T} \otimes 1:\left(\widehat{\mathcal{W}}_{G} \otimes \Omega(M)\right)_{\text {basic }} \rightarrow\left(\widehat{\mathcal{W}}_{T}^{-} \otimes \Omega(M)\right)_{\text {basic }},
$$

hence in cohomology,

$$
\mathcal{R}_{G}^{T}: \widehat{\mathcal{H}}_{G}(M) \rightarrow \widehat{\mathcal{H}}_{T}^{-}(M) .
$$

Using the horizontal projection $P_{h o r}^{\mathcal{W}}$ to identify Weil and Cartan models, the restriction map gives rise to a map $\widehat{\mathcal{C}}_{G}(M) \rightarrow \widehat{\mathcal{C}}_{T}^{-}(M)$. This map is described in the following Proposition:

Proposition 3.8. The map between Cartan models induced by the restriction $\mathcal{R}_{G}^{T}$ is the map

$$
r_{G}^{T} \otimes \exp \left(\frac{1}{2} \iota\left(r_{M}\right)\right): \widehat{\mathcal{C}}_{G}(M) \rightarrow \widehat{\mathcal{C}}_{T}^{-}(M) .
$$

Here $r_{M}=r_{a b}\left(e_{a}\right)_{M} \wedge\left(e_{b}\right)_{M}$ is the bi-vector field on $M$ corresponding to the classical $r$-matrix.

Proof. We have to compute the composition

$$
P_{\text {hor }}^{\mathcal{W}} \circ\left(r_{G}^{T} p_{G}^{T} \otimes 1\right) \circ \exp \left(\frac{1}{2} r_{a b} \iota_{a} \iota_{b} \otimes 1\right):\left(\widehat{\mathcal{W}}_{G} \otimes \Omega(M)\right)_{b a s i c} \rightarrow \widehat{\mathcal{C}}_{G}(M) .
$$

Since $\iota_{a} \otimes 1=-1 \otimes \iota_{a}$ on basic elements, the operator $\exp \left(\frac{1}{2} r_{a b} \iota_{a} \iota_{b} \otimes 1\right)$ can be replaced with $\exp \left(\frac{1}{2} r_{a b}\left(1 \otimes \iota_{a} \iota_{b}\right)\right)=\exp \left(\frac{1}{2} \iota\left(r_{M}\right)\right)$. The latter commutes with $P_{\text {hor }}^{\mathcal{W}} \circ\left(r_{G}^{T} p_{G}^{T} \otimes 1\right)$, and since $P_{h o r}^{\mathcal{W}} \circ r_{G}^{T} p_{G}^{T}=r_{G}^{T} \circ P_{h o r}^{\mathcal{W}}$ it follows that the above composition equals

$$
\left(r_{G}^{T} \otimes \exp \left(\frac{1}{2} \iota\left(r_{M}\right)\right)\right) \circ P_{h o r}^{\mathcal{W}}
$$

as claimed.

\section{Properties of the Restriction maP}

Having defined the restriction map in cohomology, some natural questions arise: Does restriction commute with quantization? Is restriction a ring map? In this Section we will give an affirmative answer to the first question and a partial answer to the second. The strategy for approaching these problems is to describe the relevant maps in terms of their integral kernels. Section 4.1 and 4.2 review material from [2].

4.1. Duality for $\widehat{W}_{G}$. The Weil differential simplifies under the isomorphism of $\widehat{W}_{G}$ given by multiplication with the function $\tau_{0} \in C^{\infty}\left(\mathfrak{g}^{*}\right) \otimes \wedge \mathfrak{g}^{*}$,

$$
\tau_{0}(\mu)=\exp \left(-\frac{1}{2} f_{a b}^{c} y^{a} y^{b} \mu_{c}\right) \text {. }
$$

Indeed,

$$
\operatorname{Ad}\left(\tau_{0}^{-1}\right) \mathrm{d}^{W}=v^{a} \iota_{a}, \quad \operatorname{Ad}\left(\tau_{0}^{-1}\right) \iota_{a}=\iota_{a}-f_{a b}^{c} \mu_{c} y^{b}
$$


These equations exhibit the Weil differential and the contraction as dual to certain operators on the space $\Omega\left(\mathfrak{g}^{*}\right)$ of differential forms on $\mathfrak{g}^{*}$. Indeed let $\langle\langle\cdot, \cdot\rangle\rangle$ denote the natural pairing between $\widehat{W}_{G}=\mathcal{E}^{\prime}\left(\mathfrak{g}^{*}\right) \otimes \wedge \mathfrak{g}^{*}$ and the space $\Omega\left(\mathfrak{g}^{*}\right)=C^{\infty}\left(\mathfrak{g}^{*}\right) \otimes \wedge \mathfrak{g}$, and define a new pairing by $\langle A, \beta\rangle:=\left\langle\left\langle\tau_{0}^{-1} A, \beta\right\rangle\right\rangle$. We denote by $\mathrm{d}^{R h}$ the de Rham differential on $\Omega\left(\mathfrak{g}^{*}\right)$ and by $\iota_{a}^{R h}$ the contractions with respect to the conjugation action.

Let $(\cdot)^{t}$ denote the transpose with respect to the pairing $\langle\cdot, \cdot\rangle$. Then Equations (20) become

$$
\mathrm{d}^{W}=-\left(\mathrm{d}^{R h}\right)^{t}, \quad \iota_{a}=-\left(\iota_{a}^{R h}+\mathrm{d} \mu_{a}\right)^{t}
$$

where the differential $\mathrm{d} \mu_{a}$ acts by multiplication on $\Omega\left(\mathfrak{g}^{*}\right)$.

The integral kernel of the identity map $\widehat{W}_{G} \rightarrow \widehat{W}_{G}$ with respect to the pairing $\langle\cdot, \cdot\rangle$ is the element

$$
\Lambda_{0}:=e^{-y^{a} d \mu_{a}} \tau_{0}(\mu) \delta_{\mu} \in\left(\widehat{W}_{G} \otimes \Omega\left(\mathfrak{g}^{*}\right)\right)^{G} .
$$

That is, $\left\langle A, \Lambda_{0}\right\rangle=A$ for any $A \in \widehat{W}_{G}$. It follows directly from (21) that

$$
\left(\mathrm{d}^{W} \otimes 1+1 \otimes \mathrm{d}^{R h}\right) \Lambda_{0}=0,\left(\iota_{a} \otimes 1+1 \otimes \iota_{a}^{R h}\right) \Lambda_{0}=-\mathrm{d} \mu_{a} \Lambda_{0} .
$$

Using $\Lambda_{0}$, various linear maps $\widehat{W}_{G} \rightarrow B$ can be described in terms of their integral kernels: For instance, the kernel to the quantization map $\mathcal{Q}: \widehat{W}_{G} \rightarrow \widehat{\mathcal{W}}_{G}$ is the element $\mathcal{Q}\left(\Lambda_{0}\right) \in \widehat{\mathcal{W}}_{G} \otimes \Omega\left(\mathfrak{g}^{*}\right)$ and the kernel of the restriction map $R_{G}^{T}: \widehat{W}_{G} \rightarrow \widehat{W}_{T}$ is the element $R_{G}^{T}\left(\Lambda_{0}\right)$.

4.2. Duality for $\widehat{\mathcal{W}}_{G}$. Just as for the commutative Weil algebra, the non-commutative Weil differential can be interpreted in terms of a duality.

Let $\theta=\theta_{a} e_{a}$ be the left invariant and $\bar{\theta}=\bar{\theta}_{a} e_{a}$ the right invariant Maurer-Cartan forms on $G$. Denote by

$$
\eta=\frac{1}{12} f_{a b c} \theta_{a} \theta_{b} \theta_{c}
$$

the canonical 3-form on $G$. It is closed, bi-invariant, and satisfies $\iota_{a} \eta=-\frac{1}{2} \mathrm{~d}\left(\theta_{a}+\bar{\theta}_{a}\right)$.

Suppose the group $G$ is a direct product of a connected, simply connected Lie group and a torus. Then the canonical embedding $\mathfrak{g} \rightarrow \mathfrak{s o}(\mathfrak{g}) \subset \mathrm{Cl}(\mathfrak{g})$ given by the adjoint action exponentiates to a map $\tau: G \rightarrow \operatorname{Spin}(\mathfrak{g}) \subset \mathrm{Cl}(\mathfrak{g})$. Explicitly, the map $\tau \in$ $C^{\infty}(G) \otimes \mathrm{Cl}(\mathfrak{g})$ is given by the formula (cf. [2] )

$$
\tau(\exp \mu)=\exp \left(-\frac{1}{2} f_{a b c} \mu_{a} x_{b} x_{c}\right) .
$$

The function $\tau$ defines an isomorphism of $\widehat{\mathcal{W}}_{G}$ by multiplication from the left. Using the symbol map, identify

$$
\widehat{\mathcal{W}}_{G} \cong \mathcal{E}^{\prime}(G) \otimes \wedge \mathfrak{g} .
$$

Using the forms $\theta_{a}$ we trivialize $T^{*} G \cong G \times \mathfrak{g}^{*}$ and identify

$$
\Omega(G) \cong C^{\infty}(G) \otimes \wedge \mathfrak{g}^{*} .
$$


Let $\langle\langle A, \beta\rangle\rangle$ be the natural pairing given by these identifications, and let $\langle A, \beta\rangle:=$ $\left\langle\left\langle\tau^{-1} A, \beta\right\rangle\right\rangle$ be the modified pairing. One finds (cf. [2], Proposition 5.6):

$$
\mathrm{d}^{\mathcal{W}}=-\left(\mathrm{d}^{R h}+\eta\right)^{t}, \quad \iota_{a}=-\left(\iota_{a}^{R h}+\frac{1}{2}\left(\theta_{a}+\bar{\theta}_{a}\right)\right)^{t}
$$

where $\mathrm{d}^{R h}, \iota_{a}^{R h}$ are de Rham differentials and contractions on the space $\Omega(G)$.

The kernel of the identity map reads

$$
\Lambda:=e^{-x_{a} \bar{\theta}_{a}} \tau(g) \delta_{g} \in \widehat{\mathcal{W}}_{G} \otimes \Omega(G),
$$

and because of (24) it has the properties,

$$
\left(\mathrm{d}^{\mathcal{W}} \otimes 1+1 \otimes \mathrm{d}^{R h}\right) \Lambda=\eta \Lambda,\left(\iota_{a} \otimes 1+1 \otimes \iota_{a}^{R h}\right) \Lambda=\frac{1}{2}\left(\theta_{a}+\bar{\theta}_{a}\right) \Lambda .
$$

As shown in Section 5 of [2], the product structure in $\widehat{\mathcal{W}}_{G}$ can be expressed in terms of the following property of the form $\Lambda$ :

$$
\Lambda^{1} \Lambda^{2}=e^{-\frac{1}{2} \theta_{a}^{1} \theta_{a}^{2}} \operatorname{Mult}_{G}^{*}(\Lambda) .
$$

Here $\Lambda^{j} \in \widehat{\mathcal{W}}_{G} \otimes \Omega(G \times G)$ are the pull-backs of $\Lambda$ to the $j$ th $G$-factor, and Mult $_{G}: G \times$ $G \rightarrow G$ is group multiplication. The quantization map $\mathcal{Q}$ has a very simple description in terms of a dual map $\Omega(G) \rightarrow \Omega(\mathfrak{g})$ : In fact, by Section 7 in [2],

$$
\mathcal{Q}=\left(e^{\varpi} \circ \exp ^{*}\right)^{t}
$$

where $\varpi \in \Omega^{2}(\mathfrak{g})^{G}$ is the image of $\eta$ under the de Rham homotopy operator $\Omega^{k}(\mathfrak{g}) \rightarrow$ $\Omega^{k-1}(\mathfrak{g})$.

4.3. Duality for $\widehat{\mathcal{W}}_{T}^{-}$. View $\delta_{t}^{-}$as a function of $t$ with values in $\mathcal{E}^{\prime}(T)^{-}$. As such it is the integral kernel of the identity map $\mathcal{E}^{\prime}(T)^{-} \rightarrow \mathcal{E}^{\prime}(T)^{-}$. The pairing between $\widehat{\mathcal{W}}_{T}$ and $\Omega(T)$ restricts to a pairing between $\widehat{\mathcal{W}}_{T}^{-}$and $\Omega(T)$. If we denote by $\theta_{k}^{T}$ the Maurer-Cartan forms for $T$, the kernel of the identity map $\widehat{\mathcal{W}}_{T}^{-} \rightarrow \widehat{\mathcal{W}}_{T}^{-}$with respect to this pairing is

$$
\Lambda_{T}^{-}:=e^{-x_{k} \theta_{k}^{T}} \delta_{t}^{-}
$$

One has the identity

$$
\left(\Lambda_{T}^{-}\right)^{1}\left(\Lambda_{T}^{-}\right)^{2}=e^{-\frac{1}{2} \theta_{k}^{T, 1} \theta_{k}^{T, 2}} \operatorname{Mult}_{T}^{*} \Lambda_{T}^{-}
$$

where $\left(\Lambda_{T}^{-}\right)^{j} \in \widehat{\mathcal{W}}_{T}^{-} \otimes \Omega(T \times T)$ are the pull-backs of $\Lambda_{T}^{-}$to the $j$ th factor, and Mult $_{T}$ : $T \times T \rightarrow T$ is the multiplication map. 
4.4. The integral kernel of the restriction map. The statement of Theorem 3.7 that $\mathcal{R}_{G}^{T}$ is a homomorphism of $T$-differential spaces can be rephrased in terms of the kernel

$$
\mathcal{R}_{G}^{T}(\Lambda) \in\left(\widehat{\mathcal{W}}_{T}^{-} \otimes \Omega(G)\right)^{T}
$$

of the restriction map:

$$
(\mathrm{d}+\eta) \mathcal{R}_{G}^{T}(\Lambda)=0, \quad\left(\iota_{k}+\frac{1}{2}\left(\theta_{k}+\bar{\theta}_{k}\right)\right) \mathcal{R}_{G}^{T}(\Lambda)=0
$$

Here we write $\mathrm{d}=\mathrm{d}^{\mathcal{W}} \otimes 1+1 \otimes \mathrm{d}^{R h}$ etc. The pull-back of $\mathcal{R}_{G}^{T}(\Lambda)$ to the maximal torus has the following decription.

Proposition 4.1. Let $\iota_{T}: T \hookrightarrow G$ be the inclusion of the maximal torus. Then

$$
\iota_{T}^{*} \mathcal{R}_{G}^{T}(\Lambda)=\Lambda_{T}^{-}
$$

Proof. We want to apply the operator $\mathcal{R}_{G}^{T}=r_{G}^{T} \otimes p_{G}^{T} \circ \mathcal{T}$ to

$$
\iota_{T}^{*} \Lambda=e^{-\sum_{k=1}^{r} x_{k} \theta_{k}^{T}} \tau(t) \delta_{t}
$$

The term $\exp \left(-\sum_{k=1}^{r} x_{k} \theta_{k}^{T}\right)$ lives in $\mathrm{Cl}(\mathfrak{t})_{\mathbb{C}} \otimes \Omega(T)$ and hence commutes with both the operator $\mathcal{T}=\exp \left(\frac{1}{2} r_{a b} \iota_{a} \iota_{b}\right)$ on $\mathrm{Cl}(\mathfrak{g})_{\mathbb{C}}$ and with the operator $p_{G}^{T}: \operatorname{Cl}(\mathfrak{g})_{\mathbb{C}} \rightarrow \mathrm{Cl}(\mathfrak{t})_{\mathbb{C}}$. Therefore,

$$
\iota_{T}^{*} \mathcal{R}_{G}^{T}(\Lambda)=e^{-\sum_{k=1}^{r} x_{k} \theta_{k}^{T}}\left(p_{G}^{T} \circ \mathcal{T}\right) \tau(t) r_{G}^{T}\left(\delta_{t}\right) .
$$

To compute $\left(p_{G}^{T} \circ \mathcal{T}\right) \tau(t)$, choose $\mu \in \mathfrak{t}$ with $t=\exp (\mu)$, and express $\tau(t)$ in the CartanWeyl basis $e_{k}, e_{\alpha}, e_{-\alpha}$. Note that since $x_{\alpha}^{2}=x_{-\alpha}^{2}=0$ and $\left[x_{\alpha}, x_{-\alpha}\right]=1$, the element $x_{\alpha} x_{-\alpha}-x_{-\alpha} x_{\alpha}$ squares to 1 . Together with $\left\langle\mu,\left[e_{\alpha}, e_{-\alpha}\right]\right\rangle=2 \pi i\langle\mu, \alpha\rangle$, this shows that

$$
\begin{aligned}
\tau(\exp \mu) & =\exp \left(i \pi \sum_{\alpha \in \mathfrak{R}_{+}}\langle\mu, \alpha\rangle\left(x_{\alpha} x_{-\alpha}-x_{-\alpha} x_{\alpha}\right)\right) \\
& =\prod_{\alpha \in \mathfrak{R}_{+}}\left(\cos (\pi\langle\mu, \alpha\rangle)+i \sin (\pi\langle\mu, \alpha\rangle)\left(x_{\alpha} x_{-\alpha}-x_{-\alpha} x_{\alpha}\right)\right)
\end{aligned}
$$

Applying the operator $p_{G}^{T} \circ \mathcal{T}=p_{G}^{T} \circ \prod_{\alpha \in \mathfrak{R}_{+}}\left(1+\frac{1}{2} \iota_{\alpha} \iota_{-\alpha}\right)$, we obtain

$$
p_{G}^{T} \circ \mathcal{T}(\tau(\exp \mu))=\prod_{\alpha \in \mathfrak{R}_{+}} \exp (i \pi\langle\mu, \alpha\rangle)=\exp (2 \pi i\langle\mu, \rho\rangle)=t^{\rho} .
$$

Finally, $t^{\rho}$ and $r_{G}^{T}\left(\delta_{t}\right)$ combine by Example 3.3, and we find

$$
\iota_{T}^{*} \mathcal{R}_{G}^{T}(\Lambda)=e^{-\sum_{k=1}^{r} x_{k} \theta_{k}^{T}} \delta_{t}^{-}=\Lambda_{T}^{-}
$$


4.5. Quantization commutes with restriction. The following result is a very useful computational tool, since it replaces the complicated restriction map $\mathcal{R}_{G}^{T}$ and the nonabelian quantization map $\mathcal{Q}_{G}$ by the much simpler restriction map $R_{G}^{T}$ and the abelian quantization map $\mathcal{Q}_{T}$.

Theorem 4.2. The diagram

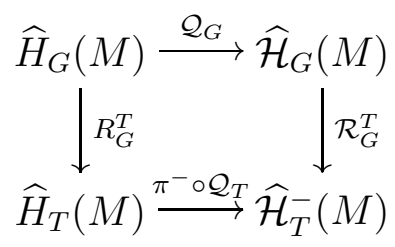

commutes.

Proof. The proof is similar to the proof in [2], Section 7, that $\mathcal{Q}$ induces a ring homomorphism in cohomology. Consider the following two homomorphisms of $T$-differential spaces, $\phi_{j}: \widehat{W}_{G} \rightarrow \widehat{\mathcal{W}}_{T}^{-}$,

$$
\phi_{1}=\pi^{-} \circ \mathcal{Q}_{T} \circ R_{G}^{T}, \quad \phi_{2}=\mathcal{R}_{G}^{T} \circ \mathcal{Q}_{G} .
$$

It suffices to show that $\phi_{1}, \phi_{2}$ are $T$-chain homotopic, that is, there exists a $T$-equivariant, odd linear map $\psi: \widehat{W}_{G} \rightarrow \widehat{\mathcal{W}}_{T}^{-}$such that $\psi$ commutes with Lie derivatives and contractions and such that $\mathrm{d}^{\mathcal{W}} \circ \psi+\psi \circ \mathrm{d}^{W}=\phi_{1}-\phi_{2}$. To construct $\psi$ we describe all maps in terms of their kernels. The kernels of the maps $\phi_{j}$ are

$$
\mathcal{K}_{j}=\left(\phi_{j} \otimes 1\right)\left(\Lambda_{0}\right) \in\left(\widehat{\mathcal{W}}_{T}^{-} \otimes \Omega\left(\mathfrak{g}^{*}\right)\right)^{T} .
$$

Since $\phi_{j}$ are homomorphisms of $T$-differential spaces, they have the property

$$
\mathrm{d} \mathcal{K}_{j}=0, \quad \iota_{k} \mathcal{K}_{j}=-\left(\mathrm{d} \mu_{k}\right) \mathcal{K}_{j} .
$$

The kernel $\mathcal{K}_{1}$ is invertible in the algebra $\widehat{\mathcal{W}}_{T}^{-} \otimes \Omega\left(\mathfrak{g}^{*}\right)$, because its form degree 0 part

$$
\left.\left(\mathcal{K}_{1}\right)_{[0]}\right|_{\mu}=\left.\phi_{1}\left(\Lambda_{0}\right)_{[0]}\right|_{\mu}=\delta_{\exp \left(\operatorname{pr}_{\mathbf{t}^{*}}(\mu)\right)}^{-}
$$

is invertible at all points $\mu \in \mathfrak{g}^{*}$. The element $\mathcal{K}_{1}^{-1} \mathcal{K}_{2}$ then has properties

$$
\iota_{k}\left(\mathcal{K}_{1}^{-1} \mathcal{K}_{2}\right)=0, \mathrm{~d}\left(\mathcal{K}_{1}^{-1} \mathcal{K}_{2}\right)=0
$$

so that $\mathcal{K}_{1}^{-1} \mathcal{K}_{2}$ is a closed element in $\left(\widehat{\mathcal{W}}_{T}^{-} \otimes \Omega\left(\mathfrak{g}^{*}\right)\right)_{\text {basic }}$. Since $\mathfrak{g}^{*}$ is equivariantly contractible, the pull-back under the inclusion $\iota:\{0\} \rightarrow \mathfrak{g}^{*}$ induces an isomorphism in cohomology. Since both $\phi_{j}$ map the identity to the identity, $\iota^{*} \mathcal{K}_{j}=1$ and therefore $\iota^{*}\left(\mathcal{K}_{1}^{-1} \mathcal{K}_{2}\right)=1$. Hence, there exists $\mathcal{N}^{\prime} \in\left(\widehat{\mathcal{W}}_{T}^{-} \otimes \Omega\left(\mathfrak{g}^{*}\right)\right)_{\text {basic }}$ with

$$
\mathcal{K}_{1}^{-1} \mathcal{K}_{2}=1-\mathrm{d} \mathcal{N}^{\prime}
$$

and letting $\mathcal{N}=\mathcal{K}_{1} \mathcal{N}^{\prime}$ and using that $\mathcal{K}_{1}$ is closed we find

$$
\mathcal{K}_{2}=\mathcal{K}_{1}-\mathrm{d} \mathcal{N} \text {. }
$$


Consequently, the linear map $\psi$ with kernel $\mathcal{N}$ is $T$-equivariant and satisfies $\mathrm{d}^{\mathcal{W}} \circ \psi+$ $\psi \circ \mathrm{d}^{W}=\phi_{1}-\phi_{2}$. Since

$$
\left(\iota_{k}+\mathrm{d} \mu_{k}\right) \mathcal{N}=0
$$

the map $\psi$ commutes with $T$-contractions.

Taking $M=\mathrm{pt}$, one recovers the fact from Lie group theory that the diagram

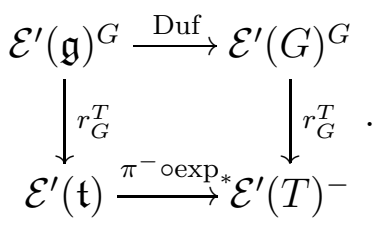

commutes.

4.6. Ring structure. It is known that all arrows in the diagram (29) are in fact ring maps: For the horizontal arrows this is the Duflo theorem, while for the vertical arrows it follows easily from the formula for the convolution of irreducible characters. This raises the question whether the arrows in the diagram (28) are ring maps as well? The left vertical arrow $R_{G}^{T}: \widehat{H}_{G}(M) \rightarrow \widehat{H}_{T}(M)$ is a ring map, and the horizontal arrows are ring maps by Theorem 8.1 in [2].

To address the problem whether the right vertical arrow $\mathcal{R}_{G}^{T}: \widehat{\mathcal{H}}_{G}(M) \rightarrow \widehat{\mathcal{H}}_{T}^{-}(M)$ is a ring map, let us consider the two maps $\phi_{j}: \widehat{\mathcal{W}}_{G} \otimes \widehat{\mathcal{W}}_{G} \rightarrow \widehat{\mathcal{W}}_{T}^{-}$defined respectively by restriction after multiplication and multiplication after restriction. The kernels $\mathcal{K}_{j} \in$ $\widehat{\mathcal{W}}_{T}^{-} \otimes \Omega(G \times G)$ for these two maps are

$$
\mathcal{K}_{1}=\mathcal{R}_{G}^{T}\left(\Lambda^{1} \Lambda^{2}\right), \quad \mathcal{K}_{2}=\mathcal{R}_{G}^{T}\left(\Lambda^{1}\right) \mathcal{R}_{G}^{T}\left(\Lambda^{2}\right)
$$

where the superscripts denote the pull-backs to the respective $G$-factor. Since $\phi_{j}$ are $T$-equivariant chain maps commuting with $T$-contractions, these kernels satisfy

$$
\left(\mathrm{d}+\eta^{1}+\eta^{2}\right) \mathcal{K}_{j}=0, \quad\left(\iota_{k}+\frac{1}{2}\left(\theta_{k}^{1}+\bar{\theta}_{k}^{1}\right)+\frac{1}{2}\left(\theta_{k}^{2}+\bar{\theta}_{k}^{2}\right)\right) \mathcal{K}_{j}=0
$$

$(k=1, \ldots, r)$. To show that $\phi_{1}, \phi_{2}$ induce the same map in cohomology, it would be enough to show that there exists $\mathcal{N} \in\left(\widehat{W}_{T}^{-} \otimes \Omega(G \times G)\right)^{T}$ with

$$
\mathcal{K}_{2}=\mathcal{K}_{1}-\left(\mathrm{d}+\eta^{1}+\eta^{2}\right) \mathcal{N}
$$

and

$$
\left(\iota_{k}+\frac{1}{2}\left(\theta_{k}^{1}+\bar{\theta}_{k}^{1}\right)+\frac{1}{2}\left(\theta_{k}^{2}+\bar{\theta}_{k}^{2}\right)\right) \mathcal{N}=0 .
$$

We do not know whether $\mathcal{N}$ with these properties exists in general, however, for our applications the following weaker statement will suffice:

Proposition 4.3. There exists a T-invariant tubular neighborhood $V$ of $T$ in $G$, and $\mathcal{N} \in\left(\widehat{\mathcal{W}}_{T}^{-} \otimes \Omega(V \times V)\right)^{T}$ such that equations (30) and (31) hold over $V \times V$. 
Proof. We begin by showing that the pull-backs of $\mathcal{K}_{1}$ and $\mathcal{K}_{2}$ under the inclusion $\iota_{T \times T}$ : $T \times T \rightarrow G \times G$ coincide. We compute $\iota_{T \times T}^{*} \mathcal{K}_{2}$ using Proposition 4.1:

$$
\iota_{T \times T}^{*} \mathcal{K}_{2}=\left(\Lambda_{T}^{-}\right)^{1}\left(\Lambda_{T}^{-}\right)^{2}=e^{-\frac{1}{2} \sum_{k=1}^{r} \theta_{k}^{1, T} \theta_{k}^{2, T}} \operatorname{Mult}_{T}^{*} \Lambda_{T}^{-}
$$

By another application of Proposition 4.1 we find

$$
\iota_{T \times T}^{*} \mathcal{K}_{1}=\iota_{T \times T}^{*} e^{-\frac{1}{2} \theta_{a}^{1} \bar{\theta}_{a}^{2}} \operatorname{Mult}_{G}^{*} \mathcal{R}_{G}^{T}(\Lambda)=e^{-\frac{1}{2} \sum_{k=1}^{r} \theta_{k}^{1, T} \theta_{k}^{2, T}} \operatorname{Mult}_{T}^{*} \Lambda_{T}^{-}
$$

Since $\iota_{T \times T}^{*} \mathcal{K}_{1}$ has non-vanishing form degree 0 part, there exists a $T$-invariant neighborhood $V$ of $T$ such that $\mathcal{K}_{1}$ has non-vanishing form degree 0 part, and is therefore invertible, on $V \times V$. We then have, over $V \times V$,

$$
\mathrm{d}\left(\mathcal{K}_{1}^{-1} \mathcal{K}_{2}\right)=0, \quad \iota_{k}\left(\mathcal{K}_{1}^{-1} \mathcal{K}_{2}\right)=0
$$

while $\iota_{T \times T}^{*}\left(\mathcal{K}_{1}^{-1} \mathcal{K}_{2}\right)=1$. Since $V$ retracts $T$-equivariantly onto $T$, the de Rham homotopy operator for the retraction $V \times V \rightarrow T \times T$ defines an element $\mathcal{N}^{\prime} \in\left(\widehat{\mathcal{W}}_{T}^{-} \otimes \Omega(V \times V)\right)_{\text {basic }}$ such that

$$
\mathcal{K}_{1}^{-1} \mathcal{K}_{2}=1-\mathrm{d} \mathcal{N}^{\prime}
$$

Letting $\mathcal{N}=\mathcal{K}_{1} \mathcal{N}^{\prime}$ this gives (30). Equation (31) follows from the equation for $\mathcal{K}_{2}, \mathcal{K}_{1}$.

We will apply this result in the following way. Suppose $\beta_{1}, \beta_{2} \in\left(\widehat{\mathcal{W}}_{G} \otimes \Omega(M)\right)_{\text {basic }}$ are two equivariant cocycles for a given $G$-manifold $M$. Suppose moreover that for some $T$-invariant open subset $U \subset M$, the restrictions $\beta_{j} \mid U$ take values in $\left(\mathcal{E}^{\prime}(V) \otimes \mathrm{Cl}(\mathfrak{g}) \otimes\right.$ $\Omega(U)$ ) where $V$ is the tubular neighborhood of $T$ as in the proposition. Then the two cocycles $\mathcal{R}_{G}^{T}\left(\beta_{1} \beta_{2}\right)$ and $\mathcal{R}_{G}^{T}\left(\beta_{1}\right) \mathcal{R}_{G}^{T}\left(\beta_{2}\right)$ in the space $\left(\widehat{\mathcal{W}}_{T}^{-} \otimes \Omega(M)\right)_{\text {basic }}$ are cohomologous if restricted to $U$.

\section{Localization formulas fOR $\widehat{\mathcal{H}}_{G}(M)$.}

In this Section we derive a new nonabelian localization formula which applies to equivariant cohomology $\widehat{\mathcal{H}}_{G}(M)$.

5.1. Localization formulas. The localization formulas discussed in this paper are derived from a common principle, Theorem 5.1 below. Let $M$ be an oriented manifold, acted upon by a compact Lie group $G$. For any $\xi \in \mathfrak{g}$ the derivation

$$
\mathrm{d}_{\xi}=\mathrm{d}-2 \pi i \iota_{\xi}: \Omega(M) \rightarrow \Omega(M)
$$

is a differential on the kernel of $L_{\xi}$ :

$$
C_{\xi}(M)=\left\{\beta \in \Omega(M), L_{\xi} \beta=0\right\} .
$$

There is a natural ring homomorphism

$$
J_{\xi}: C_{G}(M) \rightarrow C_{\xi}(M), \beta \mapsto \beta(2 \pi i \xi) .
$$

Here we view elements of $C_{G}(M)$ as polynomials on $\mathfrak{g}_{\mathbb{C}}$ with values in differential forms, and $\beta(2 \pi i \xi)$ is the evaluation of that polynomial. The localization formula expresses 
the integral of $\mathrm{d}_{\xi}$-cocycles in terms of integrals over the zeroes of the vector field $\xi_{M}$. Since $G$ is compact, $\xi_{M}^{-1}(0)$ is a union of compact, smooth, orientable submanifolds. Let $\mathcal{F}(\xi)$ denote the set of connected components of $\xi_{M}^{-1}(0)$, and for any $F \in \mathcal{F}(\xi)$ choose an orientation on $F$. Let the normal bundle $\nu_{F}$ be equipped with the orientation obtained from the orientation on $M$ and on $F$.

Let $T$ be a maximal torus of $G$ with $\xi \in \mathfrak{t}$. Then every $F$ is a $T$-invariant submanifold; let $\operatorname{Eul}\left(\nu_{F}\right) \in C_{T}(F)$ be the $T$-equivariant Euler form of $\nu_{F}$, for some choice of invariant metric and connection. We claim that

$$
J_{\xi}\left(\operatorname{Eul}\left(\nu_{F}\right)\right)=\operatorname{Eul}\left(\nu_{F}, 2 \pi i \xi\right) \in C_{\xi}(F)
$$

is invertible in $C_{\xi}(F)$. For this it suffices to note that its form degree 0 part is invertible. Up to a constant, the form degree 0 part is the Pfaffian of the endomorphism of the fibers of $\nu_{F}$ defined by $\xi$. Since $\xi_{\nu_{F}}$ vanishes precisely on $F$, this endomorphism is invertible, and therefore the Pfaffian is non-zero.

The following integration formula (32) was proved by Berline-Vergne [6] in the Cartan model of equivariant cohomology and by Atiyah-Bott [4] using the topological definition. It generalizes earlier work of Bott [8, 7] on the zeroes of holomorphic vector fields on complex manifolds and of Baum-Cheeger [5] (see also [16], Chapter II.6) on zeroes of Killing vector fields on Riemannian manifolds.

We include the proof, since the result in [6] or [4] is stated in less generality than what is needed here, although their argument extends.

Theorem 5.1 (Localization formula). Let $M$ be an oriented $G$-manifold, and $\xi \in \mathfrak{g}$. For any compactly supported $d_{\xi}$-cocycle $\gamma \in C_{\xi}(M)$, the integral of $\gamma$ over $M$ is given by the localization formula,

$$
\int_{M} \gamma=\sum_{F \in \mathcal{F}(\xi)} \int_{F} \frac{\iota_{F}^{*} \gamma}{\operatorname{Eul}\left(\nu_{F}, 2 \pi i \xi\right)}
$$

Proof. Our proof is a simple adaptation of the proof given in Guillemin-Sternberg [12], which in turn is based on the original proofs of Berline-Vergne and Atiyah-Bott. We may assume that $G=T$ is abelian.

For each $F$, let $U_{F} \cong \nu_{F}$ be a $T$-invariant tubular neighborhood of $F$. Let $f \in$ $C^{\infty}(M, \mathbb{R})$ be a function such that $f$ vanishes in a neighborhood of the fixed point set and $f=1$ in a neighborhood of $M \backslash \bigcup_{F} U_{F}$. Choose an invariant metric $g$ on $M$ and let $\psi$ be a $T$-invariant 1 -form, defined on the complement of the fixed point set, $\psi:=g\left(\xi_{M}, \cdot\right) / g\left(\xi_{M}, \xi_{M}\right)$. Then $\mathrm{d}_{\xi} \psi$ is invertible. Write

$$
\gamma=\gamma^{\prime}+\mathrm{d}_{\xi}\left(f \frac{\psi}{\mathrm{d}_{\xi} \psi} \gamma\right)
$$

Then $\int_{M} \gamma=\int_{M} \gamma^{\prime}$. The form $\gamma^{\prime}$ is supported on $\bigcup_{F} U_{F}$ and agrees with $\gamma$ near $\bigcup_{F} F$.

This reduces the proof to the case where $\gamma$ is compactly supported in $M=\nu_{F}$ for some $F$. Let $\operatorname{Th}\left(\nu_{F}\right) \in C_{T}\left(\nu_{F}\right)$ be the Mathai-Quillen form [18] representing the Tequivariant Thom class of $F$. It has compact support in fiber direction and its pull-back 
to $F$ represents the equivariant Euler form, $\operatorname{Eul}\left(\nu_{F}\right)$. Let $\operatorname{Th}\left(\nu_{F}, 2 \pi i \xi\right)=J_{\xi} \operatorname{Th}\left(\nu_{F}\right)$. Then the pull-back of $\operatorname{Eul}\left(\nu_{F}, 2 \pi i \xi\right)$ to $\pi_{F}: \nu_{F} \rightarrow F$ is $\mathrm{d}_{\xi}$-cohomologous to $\operatorname{Th}\left(\nu_{F}, 2 \pi i \xi\right)$. Consequently the quotient $\operatorname{Th}\left(\nu_{F}, 2 \pi i \xi\right) / \pi_{F}^{*} \operatorname{Eul}\left(\nu_{F}, 2 \pi i \xi\right)$ is $\mathrm{d}_{\xi}$-cohomologous to 1 . Using the main property of the Thom form that its fiber integral is 1 , and replacing $\gamma$ by the $\mathrm{d}_{\xi^{-}}$cohomologous form $\pi_{F}^{*} \iota_{F}^{*} \gamma$ we find:

$$
\begin{aligned}
\int_{\nu_{F}} \gamma & =\int_{\nu_{F}} \frac{\gamma}{\pi_{F}^{*} \operatorname{Eul}\left(\nu_{F}, 2 \pi i \xi\right)} \operatorname{Th}\left(\nu_{F}, 2 \pi i \xi\right) \\
& =\int_{\nu_{F}} \pi_{F}^{*}\left(\frac{\iota_{F}^{*} \gamma}{\operatorname{Eul}\left(\nu_{F}, 2 \pi i \xi\right)}\right) \operatorname{Th}\left(\nu_{F}, 2 \pi i \xi\right)=\int_{F} \frac{\iota_{F}^{*} \gamma}{\operatorname{Eul}\left(\nu_{F}, 2 \pi i \xi\right)}
\end{aligned}
$$

Remark 5.2. Later we need the following observation about the right hand side of the integration formula. Suppose $\beta \in C_{\xi}(F)$ is a compactly supported $T$-invariant $\mathrm{d}_{\xi}$-cocycle, and $\zeta \in \mathfrak{t}$. Then $\iota_{\zeta} \beta$ is a $\mathrm{d}_{\xi^{-c o c y c l e}} \mathrm{~d}_{\xi} \iota_{\zeta} \beta=-\iota_{\zeta} \mathrm{d}_{\xi} \beta+L_{\zeta} \beta=0$. We claim that

$$
\int_{F} \frac{\iota_{\zeta} \beta}{\operatorname{Eul}\left(\nu_{F}, 2 \pi i \xi\right)}=0
$$

As in the proof, $\pi_{F}^{*} \beta \in C_{\xi}\left(\nu_{F}\right)$ can be replaced with a compactly supported, $T$-invariant, $\mathrm{d}_{\xi^{-}}$cohomologous form $\gamma$, which agrees with $\pi_{F}^{*} \beta$ near $F$. By the localization formula, used in reverse, the above integral equals $\int_{\nu_{F}} \iota_{\zeta} \gamma$, which is 0 since $\iota_{\zeta} \gamma$ has vanishing top degree.

The localization formula is usually stated as an integration formula for cocycles in $C_{G}(M)$, taking $\gamma$ of the form $\beta(2 \pi i \xi)$ for some $\beta \in C_{G}(M)$. However, not every $\mathrm{d}_{\xi^{-}}$ cocycle arises in this way, so (32) is a more general statement. Indeed, the complexes $\widehat{C}_{G}(M)$ and $\widehat{\mathcal{C}}_{G}(M)$ produce other examples:

5.1.1. The map $J_{\xi}: \widehat{C}_{G}(M) \rightarrow C_{\xi}(M)$. For any $\xi \in \mathfrak{g}$, the map

$$
J_{\xi}: \widehat{C}_{G}(M) \rightarrow C_{\xi}(M), \beta \mapsto\left\langle\beta, e^{2 \pi i\langle\mu, \xi\rangle}\right\rangle
$$

is a chain map since

$$
\begin{aligned}
\left(\mathrm{d}_{M}-2 \pi i \iota\left(\xi_{M}\right)\right)\left\langle\beta, e^{2 \pi i\langle\mu, \xi\rangle}\right\rangle & =\left\langle\left(v^{a}-2 \pi i \xi^{a}\right) \iota_{a} \beta, e^{2 \pi i\langle\mu, \xi\rangle}\right\rangle \\
& =\left\langle\iota_{a} \beta,\left(\frac{\partial}{\partial \mu_{a}}-2 \pi i \xi^{a}\right) e^{2 \pi i\langle\mu, \xi\rangle}\right\rangle=0 .
\end{aligned}
$$

The localization formula describes the Fourier components of the integration map

$$
\int_{M}: \widehat{H}_{G}(M) \rightarrow \widehat{H}_{G}(\mathrm{pt})=\mathcal{E}^{\prime}\left(\mathfrak{g}^{*}\right)^{G}
$$


5.1.2. The map $\mathcal{J}_{\lambda}: \widehat{\mathcal{C}}_{T}(M) \rightarrow C_{\lambda}(M)$. Suppose $G=T$ is a torus, and that its Lie algebra $\mathfrak{t}$ is equipped with an inner product. For any weight $\lambda \in \Lambda^{*} \subset \mathfrak{t}$ the map

$$
\mathcal{J}_{\lambda}: \widehat{\mathcal{C}}_{T}(M) \rightarrow C_{\lambda}(M), \quad \beta \mapsto\left\langle\beta, t^{\lambda}\right\rangle
$$

is a chain map, by a calculation similar to that for $\widehat{\mathcal{C}}_{G}(M)$ :

$$
\begin{aligned}
\left(\mathrm{d}_{M}-2 \pi i \iota\left(\lambda_{M}\right)\right)\left\langle\beta, t^{\lambda}\right\rangle & =\left\langle\left(v^{a}-2 \pi i \lambda^{a}\right) \iota_{a} \beta, t^{\lambda}\right\rangle \\
& =\left\langle\iota_{a} \beta,\left(e_{a}^{L}-2 \pi i \lambda_{a}\right) t^{\lambda}\right\rangle=0 .
\end{aligned}
$$

We hence obtain a map $J_{\lambda}: \widehat{\mathcal{C}}_{T}(M) \rightarrow C_{\lambda}(M)$. In this case, the localization formula describes the Fourier coefficients $\left\langle\int_{M} \beta, t^{\lambda}\right\rangle$ of the integration map

$$
\int_{M}: \widehat{\mathcal{C}}_{T}(M) \rightarrow \widehat{\mathcal{C}}_{T}(\mathrm{pt})=\mathcal{E}^{\prime}(T) .
$$

5.1.3. The map $\mathcal{J}_{\lambda}: \widehat{\mathcal{C}}_{G}(M) \rightarrow C_{\lambda+\rho}(M)$. The map from 5.1.2 generalizes to the non-abelian case as follows. We assume that $G$ is a product of a simply connected group and a torus. The main result of Section 3 was the construction of a chain map, $\widehat{\mathcal{C}}_{G}(M) \rightarrow \widehat{\mathcal{C}}_{T}^{-}(M)$. Composing with the inclusion $\widehat{\mathcal{C}}_{T}^{-}(M) \rightarrow \widehat{\mathcal{C}}_{T}(M)$ and the map $\widehat{\mathcal{C}}_{T}(M) \rightarrow C_{\lambda+\rho}(M)$ we obtain a chain map,

$$
\mathcal{J}_{\lambda}: \widehat{\mathcal{C}}_{G}(M) \rightarrow C_{\lambda+\rho}(M), \beta \mapsto e^{\frac{1}{2} \iota\left(r_{M}\right)}\left\langle\beta, \Delta_{\lambda}\right\rangle
$$

Since the contraction operator $e^{\frac{1}{2} \iota\left(r_{M}\right)}$ does not change the top form degree part,

$$
\int_{M} \mathcal{J}_{\lambda}(\beta)=\left\langle\int_{M} \beta, \Delta_{\lambda}\right\rangle=\left(\operatorname{dim} V_{\lambda}\right)^{-1}\left\langle\int_{M} \beta, \chi_{\lambda}\right\rangle
$$

showing that the localization formula describes the Fourier coefficients of the map

$$
\int_{M}: \widehat{\mathcal{H}}_{G}(M) \rightarrow \widehat{\mathcal{H}}_{G}(\mathrm{pt})=\mathcal{E}^{\prime}(G)^{G}
$$

Applying Theorem 5.1 to this situation we have proved the main result of this paper:

Theorem 5.3. Let $\beta \in \widehat{\mathcal{C}}_{G}(M)$ be an equivariant cocycle. Then

$$
\left\langle\int_{M} \beta, \chi_{\lambda}\right\rangle=\operatorname{dim} V_{\lambda} \sum_{F \in \mathcal{F}(\lambda+\rho)} \int_{F} \frac{\iota_{F}^{*}\left(e^{\frac{1}{2} \iota\left(r_{M}\right)}\left\langle\beta, \Delta_{\lambda}\right\rangle\right)}{\operatorname{Eul}\left(\nu_{F}, 2 \pi i(\lambda+\rho)\right)} .
$$

for all $\lambda \in \Lambda_{+}^{*}$.

For the subcomplex $\mathcal{C}_{G}(M)=\left(U(\mathfrak{g})_{\mathbb{C}} \otimes \Omega(M)\right)^{G}$, the integration map takes values in the space of Casimir elements $U(\mathfrak{g})_{\mathbb{C}}^{G}$. Any Casimir element $P$ is determined by its eigenvalues in all irreducible representations, or equivalently by its traces $\operatorname{Tr}_{V_{\lambda}}(P)$.

Below we will need the following additional facts about the map $\mathcal{J}_{\lambda}$. First, suppose $\beta_{0} \in \widehat{C}_{G}(M)$ is a cocycle and that $\beta=\mathcal{Q}\left(\beta_{0}\right)$ is its quantization. Then then $\mathcal{J}_{\lambda}(\beta)$ and $J_{\lambda+\rho}\left(\beta_{0}\right)$ are cohomologous in $C_{\lambda+\rho}$. This follows from the "quantization commutes with 
restriction" Theorem, 4.2 and the definition of the maps $J_{\lambda+\rho}$ and $\mathcal{J}_{\lambda}$. Theorem 5.3 does not yield anything new for such classes.

Next, suppose $\beta \in \widehat{\mathcal{C}}_{G}(M)$ is a product of two cocycles, $\beta=\beta_{1} \odot \beta_{2}$. Suppose that both $\beta_{1}$ and $\beta_{2}$ have the property that if the support of the test function $\phi \in C^{\infty}(G)$ does not meet $T$, then $\left\langle\beta_{j}, \phi\right\rangle$ vanish near all $F \in \mathcal{F}(\lambda+\rho)$. (For example, this is trivially the case for any $\beta_{j}$ contained in $\mathcal{C}_{G}(M) \subset \widehat{\mathcal{C}}_{G}(M)$. According to the remarks at the end of Section 1.6, this means that

$$
\left(r_{G}^{T} \otimes e^{\frac{1}{2} \iota\left(r_{M}\right)}\right)\left(\beta_{1} \odot \beta_{2}\right) \quad \text { and } \quad\left(r_{G}^{T} \otimes e^{\frac{1}{2} \iota\left(r_{M}\right)}\right)\left(\beta_{1}\right) \odot\left(r_{G}^{T} \otimes e^{\frac{1}{2} \iota\left(r_{M}\right)}\right)\left(\beta_{2}\right)
$$

are cohomologous near the fixed point set. Here we are using the product $\odot$ on $\widehat{\mathcal{C}}_{T}(M)^{-}$ the product induced by the multiplication

$$
\operatorname{diag}_{M}^{*} \circ \exp \left(-\frac{1}{2} \sum_{k=1}^{r} \iota_{k}^{1} \iota_{k}^{2}\right)
$$

on $\Omega(M)^{T}$. It follows that in the localization formula, one can replace $\mathcal{J}_{\lambda}\left(\beta_{1} \odot \beta_{2}\right)$ by $\mathcal{J}_{\lambda}\left(\beta_{1}\right) \odot \mathcal{J}_{\lambda}\left(\beta_{2}\right)$. According to Remark 5.2, in the integral over $F$ we may replace $\odot$ by simply the wedge product. Hence we have shown:

Lemma 5.4. Let $M$ be a compact oriented $G$-manifold, and $\lambda \in \Lambda_{+}^{*}$. Suppose the cocycles $\beta_{1}, \beta_{2} \in \widehat{\mathcal{C}}_{G}(M)$ have the property that if the support of $\phi \in C^{\infty}(G)$ does not meet $T$, then the support of $\left\langle\beta_{j}, \phi\right\rangle$ does not meet the fixed point sets $F \in \mathcal{F}(\lambda+\rho)$. Then

$$
\left\langle\int_{M}\left(\beta_{1} \odot \beta_{2}\right), \chi_{\lambda}\right\rangle=\operatorname{dim} V_{\lambda} \sum_{F \in \mathcal{F}(\lambda+\rho)} \int_{F} \frac{\mathcal{J}_{\lambda}\left(\beta_{1}\right) \mathcal{J}_{\lambda}\left(\beta_{2}\right)}{\operatorname{Eul}\left(\nu_{F}, 2 \pi i(\lambda+\rho)\right)} .
$$

\section{ApplicAtions TO MOMENT MAPS}

In this Section we discuss applications of our localization formula to moment map theory. The main result is a Duistermaat-Heckman formula for group-valued moment maps.

6.1. Duistermaat-Heckman formula for $\mathfrak{g}^{*}$-valued moment maps. We recall the definition of a Hamiltonian $G$-space.

Definition 6.1. A Hamiltonian $G$-space is a triple $\left(M, \omega_{0}, \Phi_{0}\right)$ consisting of a $G$-space $M$, a 2 -form $\omega_{0}$, and an equivariant map $\Phi_{0} \in C^{\infty}\left(M, \mathfrak{g}^{*}\right)^{G}$ satisfying

$$
\begin{array}{cl}
\mathrm{d} \omega_{0}=0 & \text { Cocycle condition } \\
\iota\left(\xi_{M}\right) \omega_{0}=\mathrm{d}\left\langle\Phi_{0}, \xi\right\rangle & \text { Moment map condition } \\
\operatorname{ker}\left(\left(\omega_{0}\right)_{x}\right)=\{0\} & \text { Non-degeneracy }
\end{array}
$$

The first two conditions imply that the form $\omega_{0}$ is $G$-invariant. Basic examples of Hamiltonian $G$-spaces are coadjoint orbits $\mathcal{O}=G \cdot \mu$ for $\mu \in \mathfrak{g}^{*}$, with moment map the embedding $\Phi_{0}: \mathcal{O} \rightarrow \mathfrak{g}^{*}$. 
Given a Hamiltonian $G$-space $\left(M, \omega_{0}, \Phi_{0}\right)$ and a coadjoint orbit $\mathcal{O}=G \cdot \mu$ such that $\mu$ is a regular value of the moment map the reduced space $M_{\mathcal{O}}:=\Phi^{-1}(\mathcal{O}) / G$ is an orbifold with a naturally induced symplectic form $\left(\omega_{0}\right)_{\mathcal{O}}$.

For any Hamiltonian $G$-space $M$ let the equivariant Liouville form be the element of $\widehat{W}_{G} \otimes \Omega(M)$ defined as

$$
\mathcal{L}_{0}=e^{\omega_{0}} \Phi_{0}^{*} \Lambda_{0}
$$

where $\Lambda_{0} \in \widehat{\mathcal{W}}_{G} \otimes \Omega\left(\mathfrak{g}^{*}\right)$ is the integral kernel introduced in (22). Equations (34) and (23) show that $\mathcal{L}_{0}$ is a cocycle contained in the basic subcomplex $\left(\widehat{W}_{G} \otimes \Omega(M)\right)_{\text {basic }}$. The corresponding form $\mathcal{L}_{0}^{C a r}=P_{h o r}^{W}\left(\mathcal{L}_{0}\right)$ in the Cartan model is

$$
\mathcal{L}_{0}^{C a r}=e^{\omega_{0}} \delta_{\Phi_{0}} \in \widehat{C}_{G}(M) .
$$

The top degree component of its integral over $\mathfrak{g}^{*}$,

$$
\Gamma_{0}:=\left\langle\mathcal{L}_{0}^{C a r}, 1\right\rangle_{[t o p]}=\left(e^{\omega_{0}}\right)_{[t o p]}
$$

is a volume form on $M$, defining in particular a natural orientation. For a compact oriented Hamiltonian $G$-space $\left(M, \omega_{0}, \Phi_{0}\right)$ with Liouville form $\mathcal{L}_{0}$ one defines the DuistermaatHeckman measure (DH distribution) as the integral,

$$
\mathfrak{m}_{0}:=\int_{M} \mathcal{L}_{0}^{C a r} \in \mathcal{E}^{\prime}\left(\mathfrak{g}^{*}\right)^{G}
$$

The support of the distribution $\mathfrak{m}_{0}$ is contained in the image of $\Phi_{0}$ and its singular support in the set of singular values. These two properties extend to more general DH-distributions constructed from equivariant cocycles $\beta_{0} \in C_{G}(M)$

$$
\mathfrak{m}_{0}^{\beta_{0}}:=\int_{M} \beta_{0} \mathcal{L}_{0}^{C a r} \in \mathcal{E}^{\prime}\left(\mathfrak{g}^{*}\right)^{G} .
$$

The main motivation for studying the twisted $\mathrm{DH}$ distributions $\mathfrak{m}_{0}^{\beta_{0}}$ is that they encode intersection pairings on reduced spaces. More precisely, Let $\mathrm{Vol}_{\mathfrak{g}^{*}}$ be the Riemannian volume volume on $\mathfrak{g}^{*}$ for a given invariant inner product on $\mathfrak{g}$, and let $\operatorname{Vol} G$ be the corresponding Riemannian volume of the group $G$.

For any coadjoint orbit $\mathcal{O}$ in the set of regular values of $\Phi_{0}$, the value of this distribution at $\mathcal{O}$ is given by the formula

$$
\left.\frac{\mathfrak{m}_{0}^{\beta_{0}}}{\mathrm{~d} \mathrm{Vol}_{\mathfrak{g}}^{*}}\right|_{\mathcal{O}}=\frac{\operatorname{Vol} G}{k \operatorname{Vol} \mathcal{O}} \int_{M_{\mathcal{O}}} \kappa_{\mathcal{O}}\left(\beta_{0}\right) e^{\left(\omega_{0}\right)_{\mathcal{O}}}
$$

Here $k$ is the cardinality of a generic stabilizer for the $G$-action on $M, \operatorname{Vol} \mathcal{O}$ is the Liouville volume of the coadjoint orbit and $\kappa_{\mathcal{O}}: C_{G}(M) \rightarrow C\left(M_{\mathcal{O}}\right)$ is the chain map given as a composition of the pull-back map $C_{G}(M) \rightarrow C_{G}\left(\Phi_{0}^{-1}(\mathcal{O})\right)$ and the Cartan map $C_{G}\left(\Phi_{0}^{-1}(\mathcal{O})\right) \rightarrow \Omega\left(M_{\mathcal{O}}\right)$. By a theorem of Kirwan [15], the map $\kappa_{\mathcal{O}}$ induces a surjective map in cohomology. 
Applying the localization formula to $\beta_{0} \mathcal{L}_{0}^{C a r}$, and using

$$
J_{\xi}\left(\beta_{0} \mathcal{L}_{0}^{C a r}\right)=J_{\xi}\left(\beta_{0}\right) J_{\xi}\left(\mathcal{L}_{0}^{C a r}\right)=\beta_{0}(2 \pi i \xi) e^{\omega_{0}+2 \pi i\left\langle\Phi_{0}, \xi\right\rangle}
$$

one obtains the following formula for the Fourier coefficients of $\mathfrak{m}_{0}^{\beta}$ :

Proposition 6.2 (Duistermaat-Heckman formula). Let $G$ be a compact, connected Lie group, $\left(M, \omega_{0}, \Phi_{0}\right)$ a compact Hamiltonian $G$-manifold, and let $\beta_{0} \in C_{G}(M)$ be a cocycle. Then the Fourier components of the twisted DH distribution $\mathfrak{m}_{0}^{\beta_{0}}$ are given by the formula,

$$
\left\langle\mathfrak{m}_{0}^{\beta_{0}}, e^{2 \pi i\langle\mu, \xi\rangle}\right\rangle=\sum_{F \in \mathcal{F}(\xi)} e^{2 \pi i\left\langle\Phi_{0, F}, \xi\right\rangle} \int_{F} \frac{i_{F}^{*} \beta_{0}(2 \pi i \xi) e^{\omega_{0, F}}}{\operatorname{Eul}\left(\nu_{F}, 2 \pi i \xi\right)} .
$$

Here $\omega_{0, F}=\iota_{F}^{*} \omega_{0}$ and $\Phi_{0, F}=\iota_{F}^{*} \Phi_{0}$.

6.2. Group valued moment maps. In this section we prove an analogue of the Duistermaat-Heckman formula for group valued moment maps.

Definition 6.3. [1] A $G$-valued Hamiltonian $G$-space is a triple $(M, \omega, \Phi)$ consisting of a $G$-space $M$, an invariant 2-form $\omega$, and an equivariant map $\Phi \in C^{\infty}(M, G)^{G}$ satisfying the following conditions,

$$
\begin{array}{cl}
\mathrm{d} \omega=\Phi^{*} \eta & \Phi-\text { relative cocycle condition } \\
\iota_{a} \omega=\frac{1}{2} \Phi^{*}\left(\theta_{a}+\bar{\theta}_{a}\right) & \text { Moment map condition } \\
\operatorname{ker}\left(\left(\omega_{0}\right)_{x}\right)=\left\{\xi_{M}(x) \mid \operatorname{Ad}_{\Phi(x)} \xi=-\xi\right\} & \text { Minimal degeneracy condition }
\end{array}
$$

For the motivation of this definition we refer to [1]. Basic examples of Hamiltonian $G$-spaces are conjugacy classes $\mathcal{C}:=G \cdot g$ for $g \in G$, with moment map the embedding $\Phi: \mathcal{C} \rightarrow G$.

Many concepts from the theory of Hamiltonian $G$-spaces have analogues in the groupvalued setting. In particular, if $\mathcal{C} \subset G$ is a conjugacy class contained in the set of regular values of $\Phi$, the reduced space $M_{\mathcal{C}}:=\Phi^{-1}(\mathcal{C}) / G$, is an orbifold with a naturally induced symplectic form $\omega_{\mathcal{C}}$. Our aim is to extract information about intersection pairings on reduced spaces from localization formulas on $M$.

We assume that the group $G$ is a direct product of a connected, simply connected Lie group and a torus (this assumption will be lifted in Section 6.4). The equivariant Liouville form associated to a $G$-valued Hamiltonian $G$-space $(M, \omega, \Phi)$ is defined by

$$
\mathcal{L}:=e^{\omega} \Phi^{*} \Lambda \in \widehat{\mathcal{W}}_{G} \otimes \Omega(M)
$$

with $\Lambda$ as in (25). The properties (26) of $\Lambda$ imply that $\mathcal{L}$ is an equivariant cocycle in the basic subcomplex. The Liouville form in the Cartan model, $\mathcal{L}^{C a r}=P_{h o r}^{W}(\mathcal{L})$, is given by a fairly complicated expression worked out in [3]. It is also shown in [3] that, similar to the $\mathfrak{g}^{*}$-valued setting, the top degree part $\Gamma=\left\langle\mathcal{L}^{C a r}, 1\right\rangle_{[t o p]}$ is a volume form on $M$, giving rise to a natural orientation. 
Again, one can define the DH distribution $\mathfrak{m}$ as an integral over $M$ of the equivariant Liouville form

$$
\mathfrak{m}:=\int_{M} \mathcal{L}^{C a r} \in \mathcal{E}^{\prime}(G)^{G}
$$

For any cocycle $\beta \in \mathbb{C}_{G}(M)$, define more general twisted DH-distributions

$$
\mathfrak{m}^{\beta}:=\int_{M} \beta \odot \mathcal{L}^{C a r} \in \mathcal{E}^{\prime}(G)^{G} .
$$

By Proposition 5.7 of [3] $\mathfrak{m}^{\beta}$ is supported on the image of $\Phi$, and its singular support is contained in the set of singular values of $\Phi$. The relationship between intersection pairings on the reduced space $M_{\mathcal{C}}$, for $\mathcal{C}$ contained in the set of regular values, and twisted DH distributions $\mathfrak{m}^{\beta}$ is as follows, (cf. [3]):

$$
\left.\frac{\mathfrak{m}^{\beta}}{\mathrm{d} \operatorname{Vol}_{G}}\right|_{\mathcal{C}}=\frac{\operatorname{Vol} G}{k \operatorname{Vol} \mathcal{C}} \int_{M_{\mathcal{C}}} \kappa_{\mathcal{C}}(\beta) e^{\omega_{\mathcal{C}}} .
$$

Here $\mathrm{d} \mathrm{Vol}_{G}$ is the Haar measure on $G$ defined by the inner product on $\mathfrak{g}, \operatorname{Vol} \mathcal{C}$ is the Liouville volume of the conjugacy class $\mathcal{C}$ with respect to the volume form $\Gamma_{\mathcal{C}}$, and $k$ the number of elements in a generic stabilizer. The map $\kappa_{\mathcal{C}}: \mathcal{C}_{G}(M) \rightarrow \Omega\left(M_{\mathcal{C}}\right)$ is the composition of pull-back to $\Phi^{-1}(\mathcal{C})$, the isomorphism $\mathcal{C}_{G}\left(\Phi^{-1}(\mathcal{C})\right) \cong C_{G}\left(\Phi^{-1}(\mathcal{C})\right)$ given by the inverse of the quantization map, and the Cartan map $C_{G}\left(\Phi^{-1}(\mathcal{C})\right) \rightarrow M_{\mathcal{C}}$.

6.3. DH formula for group valued moment maps. Similar to the case of Hamiltonian $G$-spaces, the Fourier decomposition of the DH-distributions $\mathfrak{m}^{\beta}$ can be obtained from a localization formula. To state the result, note first that for any $\lambda \in \Lambda_{+}^{*}$, all $F \in \mathcal{F}(\lambda+\rho)$ are contained in the pre-image of the maximal torus $\Phi^{-1}(T)$, since $\Phi$ is equivariant and since the vector field for the conjugation action $(\lambda+\rho)_{G}$ vanishes exactly on $T$. Let $\omega_{F}=\iota_{F}^{*} \omega$ and $\Phi_{F}=\iota_{F}^{*} \Phi$. Since $\Phi_{F}$ takes values in $T$, we can compose with the map $T \rightarrow S^{1}, t \mapsto t^{\lambda+\rho}$ and this composition is denoted $\left(\Phi_{F}\right)^{\lambda+\rho}$. Indeed, $F$ is a group valued Hamiltonian $T$-space with $\omega_{F}$ as 2 -form and $\Phi_{F}$ as moment map. From the fact that $(\lambda+\rho)_{M}$ vanishes on $F$, one deduces that $\left(\Phi_{F}\right)^{\lambda+\rho}$ is constant along $F$.

Theorem 6.4 (DH formula for group-valued moment maps). Let $G$ be a direct product of a connected, simply-connected Lie group and a torus, $(M, \omega, \Phi)$ a compact $G$-valued Hamiltonian $G$-space, and $\beta=\mathcal{Q}\left(\beta_{0}\right) \in C_{G}(M)$ be an equivariant cocycle. The Fourier coefficients of the twisted DH distribution $\mathfrak{m}^{\beta}$ are given by the formula,

$$
\left\langle\mathfrak{m}^{\beta}, \chi_{\lambda}\right\rangle=\operatorname{dim} V_{\lambda} \sum_{F \in \mathcal{F}(\lambda+\rho)}\left(\Phi_{F}\right)^{\lambda+\rho} \int_{F} \frac{\iota_{F}^{*} \beta_{0}(2 \pi i(\lambda+\rho))}{\operatorname{Eul}\left(\nu_{F}, 2 \pi i(\lambda+\rho)\right)} e^{\omega_{F}} .
$$

Proof. We apply the localization formula to the class $\beta \odot \mathcal{L}^{\text {Car }}$. Note that the cocycles $\beta$ and $\mathcal{L}^{C a r}$ satisfy the condition from Lemma 5.4, which therefore gives

$$
\left\langle\mathfrak{m}^{\beta}, \chi_{\lambda}\right\rangle=\operatorname{dim} V_{\lambda} \sum_{F \in \mathcal{F}(\lambda+\rho)} \int_{F} \frac{\mathcal{J}_{\lambda}(\beta) \mathcal{J}_{\lambda}\left(\mathcal{L}^{\text {Car }}\right)}{\operatorname{Eul}\left(\nu_{F}, 2 \pi i(\lambda+\rho)\right)} .
$$


By the discussion preceding Lemma 5.4, $\mathcal{J}_{\lambda}(\beta)$ may be replaced with

$$
J_{\lambda+\rho}\left(\beta_{0}\right)=\beta_{0}(2 \pi i(\lambda+\rho)) .
$$

It remains to compute $\iota_{F}^{*} \mathcal{J}_{\lambda}\left(\mathcal{L}^{C a r}\right)$. For this we we use the description of the Liouville form in the Weil model. By Proposition 4.1 we have

$$
\iota_{F}^{*} \mathcal{R}_{G}^{T}(\mathcal{L})=e^{\omega_{F}}\left(\iota_{T} \circ \Phi_{F}\right)^{*} \mathcal{R}_{G}^{T}(\Lambda)=e^{\omega_{F}} \Phi_{F}^{*} \Lambda_{T}^{-} .
$$

Therefore, the image of $\mathcal{L}^{C a r}$ under the map $\widehat{\mathcal{C}}_{G}(M) \rightarrow \widehat{\mathcal{C}}_{T}(M)^{-}$is given by $e^{\omega_{F}} \delta_{\Phi_{F}}^{-}$and we obtain

$$
\iota_{F}^{*} \mathcal{J}_{\lambda}\left(\mathcal{L}^{C a r}\right)=\left(\Phi_{F}\right)^{\lambda+\rho} e^{\omega_{F}} .
$$

Now we combine the localization formula (43) and formula (42) and use the inverse Fourier transform on the group $G$ to obtain a formula for intersection pairings on reduced spaces,

$$
\begin{aligned}
\int_{M_{\mathcal{C}}} & \kappa_{\mathcal{C}}(\beta) e^{\omega_{\mathcal{C}}} \\
& =k \operatorname{Vol} \mathcal{C} \sum_{\lambda} \frac{\chi_{\lambda}\left(\mathcal{C}^{-1}\right) \operatorname{dim} V_{\lambda}}{(\operatorname{Vol} G)^{2}} \sum_{F \in \mathcal{F}(\lambda+\rho)}\left(\Phi_{F}\right)^{\lambda+\rho} \int_{F} \frac{\iota_{F}^{*} \beta_{0}(2 \pi i(\lambda+\rho))}{\operatorname{Eul}\left(\nu_{F}, 2 \pi i(\lambda+\rho)\right)} e^{\omega_{F}},
\end{aligned}
$$

where $\chi_{\lambda}\left(\mathcal{C}^{-1}\right)$ is the value of the character $\chi_{\lambda}$ on the conjugacy class $\mathcal{C}^{-1}$.

6.4. Generalization to arbitrary compact $G$. Up to this point we made the assumption that the compact, connected group $G$ be a direct product of a simply connected group and a torus. We will now remove this assumption and show that if appropriately interpreted, the Duistermaat-Heckman formula (43) holds in the general case.

Without this assumption the definition of the Liouville form breaks down, since the form $\Lambda$ is no longer a well-defined element of $\widehat{W}_{G} \otimes \Omega^{*}(G)$. Indeed, in general a Hamiltonian $G$-manifold with group-valued moment map need not be orientable. The simplest counter-example is the conjugacy class in the rotation group $\mathrm{SO}(3)$ consisting of rotations by an angle $\pi$, which is isomorphic to $\mathbb{R} P^{2}$.

Choose a finite covering $\mathfrak{c}_{G}: G^{\prime} \rightarrow G$ such that $G^{\prime}$ is a direct product of a compact, connected, simply-connected group and a torus. The kernel $R:=\mathfrak{c}_{G}^{-1}(e)$ is a subgroup of the center of $G^{\prime}$. Given a group-valued Hamiltonian $G$-space $(M, \omega, \Phi)$, consider the fiber product,

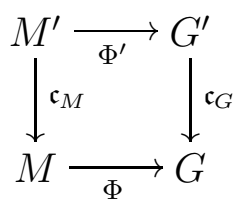

that is $M^{\prime}=\left\{\left(x, g^{\prime}\right) \in M \times G^{\prime} \mid \Phi(x)=\mathfrak{c}_{G}\left(g^{\prime}\right)\right\}$. The diagonal action of $G^{\prime}$ on $M \times G^{\prime}$ (which in fact is a $G$-action since $R$ acts trivially) leaves $M^{\prime}$ invariant, and commutes 
with the action of $R$ by deck transformations. Let $\omega^{\prime}=\mathfrak{c}_{M}^{*} \omega$. Then $\left(M^{\prime}, \omega^{\prime}, \Phi^{\prime}\right)$ is a group-valued Hamiltonian $G^{\prime}$-space. The form $\omega^{\prime}$ is invariant with respect to deck transformations, while the moment map $\Phi^{\prime}$ satisfies $c^{*} \Phi^{\prime}=c \Phi$ for $c \in R$. Given a regular value $g^{\prime} \in G^{\prime}$ of $\Phi^{\prime}$, the image $g=\mathfrak{c}_{G}\left(g^{\prime}\right) \in G$ is a regular value for $\Phi$ and there is a canonical isomorphism $M_{g^{\prime}}^{\prime}=M_{g}$.

Suppose $\beta \in \mathcal{C}_{G}(M)$ is an equivariant cocycle, and $\beta^{\prime}=\mathfrak{c}_{M}^{*} \beta \in \mathcal{C}_{G^{\prime}}\left(M^{\prime}\right)$. The form $\beta^{\prime}$ defines a twisted DH-distribution $\left(\mathfrak{m}^{\prime}\right)^{\beta^{\prime}} \in \mathcal{E}^{\prime}\left(G^{\prime}\right)^{G^{\prime}}$, invariant under the action of $R$. Let a twisted DH-distribution for $M$ be defined by

$$
\mathfrak{m}^{\beta}:=\frac{1}{\# R}\left(\mathfrak{c}_{M}\right)_{*}\left(\mathfrak{m}^{\prime}\right)^{\beta^{\prime}} \in \mathcal{E}^{\prime}(G)^{G}
$$

It is shown in [3] that formula (42), relating the values of $\mathfrak{m}^{\beta}$ and intersection pairings on reduced spaces, carries over to this more general situation.

The Fourier coefficients of $\mathfrak{m}^{\beta}$ can be read off from those for $\left(\mathfrak{m}^{\prime}\right)^{\beta^{\prime}}$. Given a dominant weight $\lambda \in \Lambda_{+}^{*}$, the corresponding irreducible character $\chi_{\lambda}^{\prime}$ for $G^{\prime}$ is the pull-back $\mathfrak{c}_{G}^{*} \chi_{\lambda}$, hence

$$
\left\langle\mathfrak{m}^{\beta}, \chi_{\lambda}\right\rangle=\frac{1}{\# R}\left\langle\left(\mathfrak{m}^{\prime}\right)^{\beta^{\prime}}, \chi_{\lambda}^{\prime}\right\rangle
$$

The pairing on the right hand side is given by the localization formula (43). For any fixed point manifold $F \in \mathcal{F}(\lambda+\rho)$ let $F^{\prime}=\mathfrak{c}_{M}^{-1}(F)$ be its pre-image in $M^{\prime}$. The union of all $F^{\prime}$ is the fixed point set for $(\lambda+\rho)_{M^{\prime}}$. Therefore, (43) shows that

$$
\left\langle\mathfrak{m}^{\beta}, \chi_{\lambda}\right\rangle=\frac{\operatorname{dim} V_{\lambda}}{\# R} \sum_{F \in \mathcal{F}(\lambda+\rho)}\left(\Phi^{\prime}\right)^{\lambda+\rho} \int_{F^{\prime}} \frac{\iota_{F^{\prime}}^{*}\left(\beta^{\prime}(2 \pi i(\lambda+\rho)) e^{\omega^{\prime}}\right.}{\operatorname{Eul}\left(\nu_{F^{\prime}}, 2 \pi i(\lambda+\rho)\right)} .
$$

We want to replace integrals over $F^{\prime}$ by integrals over $F$. Note first that $\left(\Phi_{F^{\prime}}^{\prime}\right)^{\lambda}$ is just the pull-back of $\Phi_{F}^{\lambda}$, and that $\iota_{F^{\prime}}^{*} \omega$ is the pull-back of $\iota_{F}^{*} \omega$. On the other hand, the locally constant function $\left(\Phi_{F^{\prime}}^{\prime}\right)^{\rho}: F^{\prime} \rightarrow S^{1}$ does not descend to $F$ since for $c \in R$,

$$
c^{*}\left(\Phi_{F^{\prime}}^{\prime}\right)^{\rho}=c^{\rho}\left(\Phi_{F^{\prime}}^{\prime}\right)^{\rho} \text {. }
$$

Since $2 \rho \in \Lambda^{*}$ is a weight for $G, c^{2 \rho}=1$ so that $c^{\rho}= \pm 1$ is just a sign. As shown in Section 8 of [3], this sign also gives the change in orientation of $M^{\prime}$ under the action of c. It follows that the quotient

$$
\frac{\left(\Phi^{\prime}\right)_{F^{\prime}}^{\rho}}{\operatorname{Eul}\left(\nu_{F^{\prime}}, 2 \pi i(\lambda+\rho)\right)}
$$

is invariant under $R$, and therefore descends to a form on $F$. If we denote this form by

$$
\frac{\Phi_{F}^{\rho}}{\operatorname{Eul}\left(\nu_{F}, 2 \pi i(\lambda+\rho)\right)}
$$

then (43) holds for any compact, connected Lie group. (Put differently, while $\nu_{F}$ need not be orientable, the Euler form makes sense as a form with values in the orientation bundle of $\nu_{F}$, and $\Phi_{F}^{\rho}$ descends to a section of the orientation bundle. Their quotient is a form in the usual sense.) 


\section{REFERENCES}

1. A. Alekseev, A. Malkin, and E. Meinrenken, Lie group valued moment maps, J. Differential Geom. 48 (1998), no. 3, 445-495.

2. A. Alekseev and E. Meinrenken, The non-commutative Weil algebra, Preprint, Uppsala University, University of Toronto, 1999.

3. A. Alekseev, E. Meinrenken, and C. Woodward, Duistermaat-Heckman distributions for Lie group valued moment maps, Preprint, 1999.

4. M. F. Atiyah and R. Bott, The moment map and equivariant cohomology, Topology 23 (1984), no. $1,1-28$.

5. P. Baum and J. Cheeger, Infinitesimal isometries and Pontryagin numbers, Topology 8 (1969), 173-193.

6. N. Berline and M. Vergne, Zéro d'un champ de vecteurs et classes caractéristiques équivariantes, Duke Math. J. 50 (1983), 539-549.

7. R. Bott, A residue formula for holomorphic vector-fields, J. Differential Geometry 1 (1967), 311-330.

8. _ Vector fields and characteristic numbers, Mich. Math. J. 14 (1967), 231-244.

9. H. Cartan, Notions d'algèbre différentielle; application aux groupes de Lie et aux variétés où opère un groupe de Lie., Colloque de topologie (espaces fibrés) (Bruxelles), Georges Thone, Liège, Masson et Cie., Paris, 1950.

10. M. Duflo, Opérateurs différentiels bi-invariants sur un groupe de Lie, Ann. Sci. École Norm. Sup. 10 (1977), 265-288.

11. F. G. Friedlander, Introduction to the theory of distributions, Cambridge University Press, 1982.

12. V. Guillemin and S. Sternberg, Supersymmetry and equivariant de Rham theory, Springer Verlag, 1999.

13. L. Hörmander, The analysis of linear partial differential operators I, second ed., Grundlehren der mathematischen Wissenschaften, vol. 256, Springer-Verlag, Berlin-Heidelberg-New York, 1990.

14. L. C. Jeffrey and F. C. Kirwan, Intersection theory on moduli spaces of holomorphic bundles of arbitrary rank on a Riemann surface, Ann. of Math. (2) 148 (1998), no. 2, 109-196.

15. F. C. Kirwan, Cohomology of quotients in symplectic and algebraic geometry, Mathematical Notes, vol. 31, Princeton Univ. Press, Princeton, 1984.

16. S. Kobayashi, Transformation groups in differential geometry, Ergebnisse der Mathematik und ihrer Grenzgebiete, Springer, 1972.

17. B. Kostant, Clifford algebra analogue of the Hopf-Koszul-Samelson theorem, the $\rho$-decomposition $\mathrm{C}(\mathfrak{g})=$ End $V_{\rho} \otimes \mathrm{C}(\mathrm{P})$, and the $\mathfrak{g}$-module structure of $\bigwedge \mathfrak{g}$., Adv. in Math. 125 (1997), no. 2, $275-350$.

18. V. Mathai and D. Quillen, Thom classes, superconnections, and equivariant differential forms, Topology 25 (1986), 85-106.

19. E. Witten, Two-dimensional gauge theories revisited, J. Geom. Phys. 9 (1992), 303-368.

Institute for Theoretical Physics, Uppsala University, Box 803, S-75108 Uppsala, SWEDEN

E-mail address: alekseev@teorfys.uu.se

University of Toronto, Department of Mathematics, 100 St George Street, Toronto, Ontario M5R3G3, Canada

E-mail address: mein@math.toronto.edu

Mathematics - Hill Center, Rutgers University, 110 Frelinghuysen Road, Piscataway NJ 08854-8019, USA

E-mail address: ctw@math.rutgers.edu 